\title{
2. ICHTHYOLITH BIOSTRATIGRAPHY OF DEEP-SEA CLAYS FROM THE SOUTHWESTERN HAWAIIAN ARCH ${ }^{1}$
}

\author{
John V. Firth ${ }^{2}$ and Donna Meyerhoff Hull ${ }^{3}$
}

\begin{abstract}
Sixty-nine ichthyolith taxa have been identified in otherwise unfossiliferous red clays from Cores $136-842 \mathrm{~B}-3 \mathrm{H},-4 \mathrm{H}$, and $-5 \mathrm{H}$ at Ocean Drilling Program Site 842 on the Hawaiian Arch. Based on correlation with previous studies of ichthyoliths, these assemblages indicate that sediments from 21.53 to $31.10 \mathrm{mbsf}$ are of early Miocene age. Ichthyolith-bearing sediments below this interval, from 31.10 to $35.57 \mathrm{mbsf}$, are Oligocene to early Miocene in age, whereas overlying clays from 21.53 to 19.05 mbsf are early to middle Miocene in age. Reworked ichthyoliths of Cretaceous to Eocene age are present in the cores, suggesting an exposed source of sediments of these ages during the early Miocene.
\end{abstract}

\section{INTRODUCTION}

The major objective of Leg 136 of the Ocean Drilling Program (ODP) was to drill a test site for developing ocean bottom seismometers that would eventually be placed around the Earth's oceans as the Ocean Seismographic Network. Secondary objectives were to recover the deep sea sediments and ocean crust at this site, located about 100 miles southwest of Hawaii (Fig. 1), and to study the nature and history of the crust through which the nearby Hawaiian volcanic chain emerged. The sediments consist of about $35 \mathrm{~m}$ of clays and silty clays with variable amounts of ash, radiolarians, and sponge spicules, overlying about $200 \mathrm{~m}$ of claystones and chert with some intercalated nannofossil ooze. The base of the sedimentary sequence, just overlying basaltic basement, consists of limestone (Dziewonski, Wilkens, Firth, et al., 1992). In Hole 842B, the middle and lower parts of Core $136-842 \mathrm{~B}-3 \mathrm{H}$, and Cores 136-842B-4H and -5H (from 19.05 to 35.57 meters below seafloor [mbsf]) contain dark reddish brown clays with altered volcanic ash layers. These sediments were barren of all microfossils except for ichthyoliths. A preliminary ichthyolith age determination of the cores based on two core-catcher samples was made on ship (Dziewonski, Wilkens, Firth, et al., 1992). This paper presents a more detailed analysis of the ichthyolith assemblages recovered from these cores and their significance for determining the age of the ash-bearing clays.

\section{METHODS}

Samples of $10-15 \mathrm{~cm}^{3}$ were disaggregated by boiling for $2-4 \mathrm{~min}$ in a 50:50 mixture of water and hydrogen peroxide, with Calgon added as a clay-dispersant. After boiling, samples were sieved and gently washed through 60,100 , and 230 mesh stainless steel sieves. Residues from each sieve were collected into filter paper and dried in a laboratory oven. Ichthyoliths were picked from the residues and mounted on glass slides, with cover slips fastened with Norland Optical Adhesive. The slides were studied using transmission light microscopy at $50 \times$ magnification.

Samples were not weighed before processing; therefore, we could not determine absolute abundances of the different taxa. In some samples, ichthyoliths were too abundant to pick all of them. There-

\footnotetext{
'Wilkens, R.H., Firth, J., Bender, J., et al., 1993.Proc. ODP, Sci. Results, 136: College Station, TX (Ocean Drilling Program).

${ }^{2}$ Ocean Drilling Program, Texas A\&M University Research Park, 1000 Discovery Drive, College Station, TX 77845-9547, U.S.A.

${ }^{3}$ Program in Geosciences, The University of Texas at Dallas, P.O. Box 830688. Richardson, TX 75083-0688, U.S.A.
}

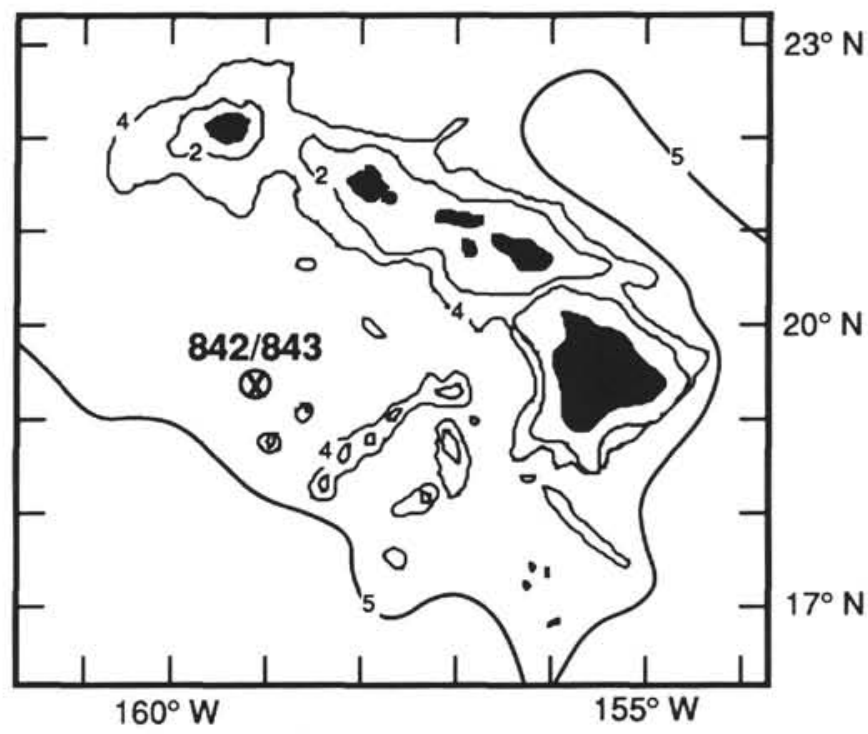

Figure 1. Location map showing Sites 842 and 843 drilled southwest of Hawaii on the Hawaiian Arch. Bathymetry in thousands of meters.

fore, we concentrated on collecting just the presence/absence data for all previously described taxa found in each sample, as well as for a few unnamed forms. After producing an initial range chart, the sample residues were reexamined to fill in and double check the ranges of identified forms, especially of taxa whose published stratigraphic ranges are useful for determining the age of these sediments.

The system of nomenclature developed by Doyle et al. (1974) and modified by Dunsworth et al. (1975), Ramsey et al. (1976), Doyle et al. (1978), Doyle and Riedel (1979b), Tway (1979), Gottfried et al. (1984a), Doyle and Riedel (1985b), Tway et al. (1985), and Winfrey et al. (1987) was followed in this paper.

\section{RESULTS}

The ranges of 69 taxa of ichthyoliths in Cores $842 \mathrm{~B}-3 \mathrm{H},-4 \mathrm{H}$, and $-5 \mathrm{H}$ are documented in Table 1 . Of these, 5 taxa are considered to be reworked, whereas several other taxa may be either reworked or have extensions of their previously reported ranges. Five undescribed taxa are also documented. Many undescribed forms are not included in the results discussed in this paper. In fact, a large fraction of the assemblages in residue is composed of undescribed forms. Of the previously 


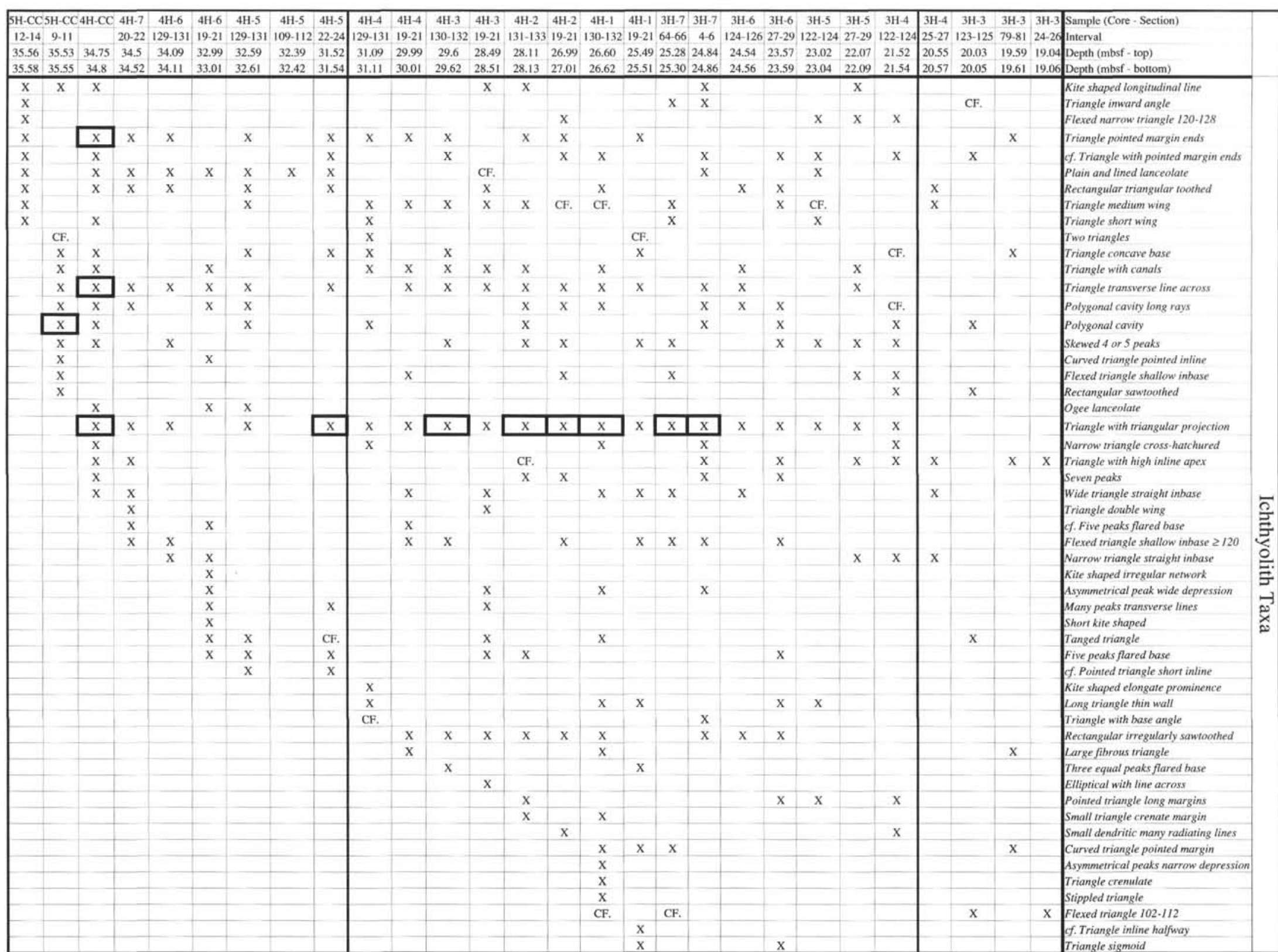

Notes: " $\mathrm{X}$ " indicates presence; "CF" indicates the presence of a comparable form to the listed taxon. Bold boxes mark where taxa have 5 or more specimens in a particular sample. Maximum previously reported age ranges of each named taxa are listed in Table 2, along with references used. 


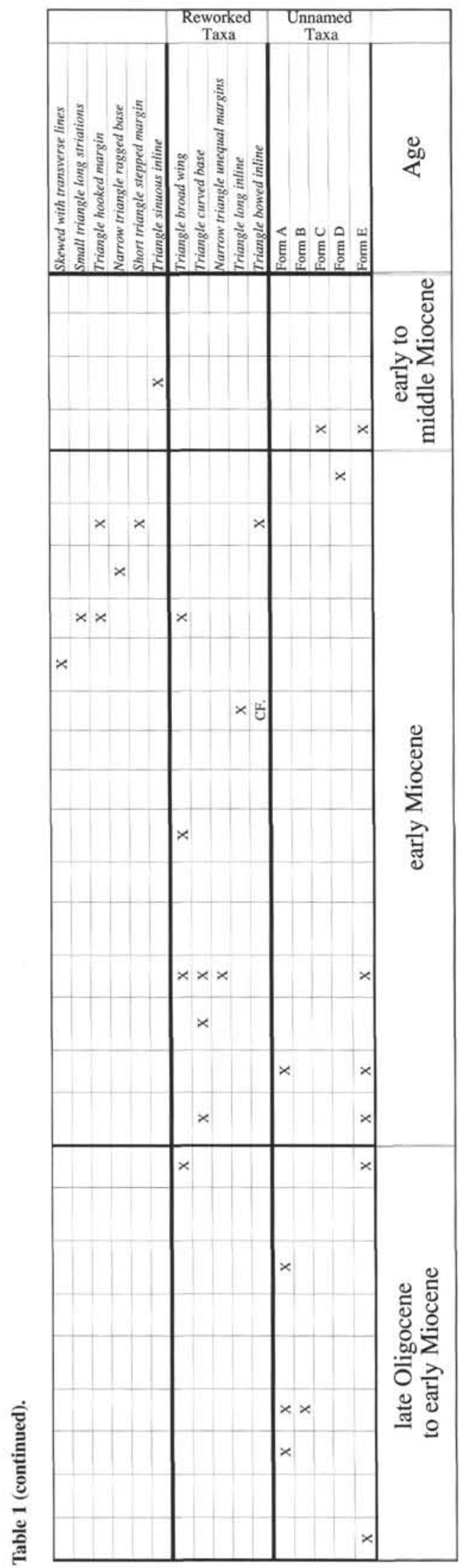

described taxa identified in our samples, only a few forms appear to be common, whereas the rest of the taxa are represented by less than 5 specimens per sample, and typically only one or two specimens per sample. Of the more common forms, Triangle with triangular projection is the most common, with 5 or more specimens per sample in 8 samples. Other forms that have 5 or more specimens per sample are Polygonal cavity, Triangle transverse line across, and Triangle pointed margin ends. The samples in which these forms are common are indicated on Table 1 by bold boxes around their occurrences. The following samples have poor preservation and/or few $(<100)$ total specimens found in the residues: $136-842 \mathrm{~B}-4 \mathrm{H}-6,129-131 \mathrm{~cm} ;-4 \mathrm{H}-$ $5,109-112 \mathrm{~cm} ;-3 \mathrm{H}-3,123-125 \mathrm{~cm} ;-3 \mathrm{H}-3,79-81 \mathrm{~cm}$; and $-3 \mathrm{H}-3$, 24-26 cm. All other samples have several hundred specimens, and have good preservation.

Because of the presence of reworked forms in the cores, only first occurrences of index taxa are considered reliable for age determinations. Most ichthyolith taxa are long-ranging, and because of the absence of other microfossil groups, only broad age assignments can be established for these cores. Age determinations were based on the cumulative age ranges of each taxa published in the extensive DSDP/ ODP and journal literature (Table 2).

Based on the successive first occurrences of Two triangles (sensu Doyle et al., 1974), Stippled triangle, Small triangle long striations, Narrow triangle ragged base, and Short triangle stepped margin, the interval from Sample 842B-4H-4, 129-131 cm (31.10 mbsf), to Sample $136-842 \mathrm{~B}-3 \mathrm{H}-4,122-124 \mathrm{~cm}(21.53 \mathrm{mbsf})$, is of early Miocene age (Fig. 2).

The interval from Section 136-842B-5H-CC to Sample 136-842B$4 \mathrm{H}-5,109-112 \mathrm{~cm}$ (32.40 mbsf), lacks taxa that are exclusively indicative of Miocene or younger age. One specimen referred to herein as cf. Two triangles Doyle et al. (1974) occurs in Sample 136-842B-5HCC, $9-11 \mathrm{~cm}$ (35.54 mbsf). This specimen possesses two closely spaced triangular projections with a well-developed inline that closely parallels the outer margin. The original description (Doyle et al., 1974, p. 845) describes this form as "two simply triangular projections, widely spaced, arising from a short, stubby base." No reference to the character of the inline is mentioned, and all illustrated specimens in Doyle et al. (1974) and Doyle and Riedel (1985a) either lack an inline or possess an inline in only one triangular projection. If we consider our specimen to fit within the original definition of this taxon, the interval from Sample 136-842B-5H-CC, 9-11 cm, to Sample 136$842 \mathrm{~B}-4 \mathrm{H}-5,22-24 \mathrm{~cm}$, may be assigned to the lower Miocene (based on range reported in Doyle and Riedel, 1985a). Two other possibilities exist: (1) the range of Two triangles may be extended into the Oligocene, or (2) this specimen does not fall within the morphotypic range of Two triangles. In either case, the age of Sample 136-842B-5H-CC, 9-11 cm, and of the lower part of Core 136-842B-4H may be restricted to the late Oligocene or younger based on the occurrences of Skewed 4 or 5 peaks and Asymmetrical peak wide depression (Tables 1 and 2). Based on the occurrence of Flexed triangle 120-128, the age of Sample 136-842B-5H-CC, 12-14 cm, may be early Oligocene or younger (Doyle and Riedel, 1985a), or late Eocene or younger (Kozarek and Orr, 1979).

The last occurrences of Skewed 4 or 5 peaks and Triangle short wing in Sections 136-842B-3H-5 and -3H-4 may indicate an age of middle Miocene above this level (based on reported ranges from Kozarek and Orr, 1979, and Doyle and Riedel, 1985a, respectively). Because of scattered occurrences of these taxa throughout the cores, and because of reworking evident by the presence of older forms, these last occurrences may not be reliable. Therefore, the interval from Samples 136-842B-3H-4, 25-27 cm (20.56 mbsf), to -3H-3, $24-26 \mathrm{~cm}$ ( $19.05 \mathrm{mbsf})$ is assigned an early to middle Miocene age.

The following taxa are reworked: Triangle broad wing, Triangle curved base, Narrow triangle unequal margins, Triangle long inline and Triangle bowed inline. These forms range in age from Upper Cretaceous to Eocene (Table 2), which indicates the exposure of older 
Table 2. List of taxa and their previously reported age ranges and references.

\begin{tabular}{|c|c|c|}
\hline Ichthyolith Taxa & Age range & References \\
\hline Kite shaped longitudinal line & late Eocene-early Oligocene & Doyle et al., 1974 \\
\hline Triangle inward angle & middle Eocene-early middle Miocene & Gottfried et al., 1984a \\
\hline Flexed narrow triangle $120-128$ & early Oligocene-Holocene & Doyle and Riedel, 1985a \\
\hline Triangle pointed margin ends & Paleocene-early middle Miocene & Doyle and Riedel, $1985 \mathrm{a}$ \\
\hline Plain and lined lanceolate & Cretaceous-middle Miocene & Winfrey et al., 1987 and Kozarek and Orr, 1979 \\
\hline Rectangular triangular toothed & Cretaceous-Pliocene & Kozarek and Orr, 1979 \\
\hline Triangle medium wing & early Paleocene-middle Miocene & Doyle and Riedel, $1985 \mathrm{a}$ \\
\hline Triangle short wing & middle Paleocene-early middle Miocene & Doyle and Riedel, $1985 \mathrm{a}$ \\
\hline Two triangles & early Miocene-Holocence & Doyle and Riedel, $1985 \mathrm{a}$ \\
\hline Triangle concave base & Paleocene/early Eocene-middle Miocene & Kozarek and Orr, 1979 \\
\hline Triangle with canals & Cretaceous-Pliocene & Kozarek and Orr, 1979 \\
\hline Triangle transverse line across & Cretaceous-Pliocene & Kozarek and Orr, 1979 \\
\hline Polygonal cavity long rays & late Eocene-early Miocene & Kozarek and Orr, 1979 \\
\hline Polygonal cavity & Cretaceous?-early Miocene & Kozarek and Orr, 1979 \\
\hline Skewed 4 or 5 peaks & late Oligocene-early Miocene & Kozarek and Orr, 1979 \\
\hline Curved triangle pointed inline & early Eocene-late Pliocene & Tway et al., 1985 \\
\hline Flexed triangle shallow inbase & Cretaceous-Holocene & Kozarek and Orr, 1979 \\
\hline Rectangular sawtoothed & Oligocene-Holocene & Doyle and Riedel, 1985a \\
\hline Ogee lanceolate & early Eocene-middle Miocene & Tway et al., 1985 \\
\hline Triangle with triangular projection & Cretaceous-Holocene & Kozarek and Orr, 1979 \\
\hline Narrow triangle cross-hachured & earliest Paleocene-middle Miocene & Kozarek and Orr, 1979 \\
\hline Triangle with high inline apex & early Oligocene-Holocene & Doyle and Riedel, 1985a \\
\hline Seven peaks & late Eocene-latest Oligocene & Kozarek and Orr, 1979 \\
\hline Wide triangle straight inbase & early Eocene-Holocene & Kozarek and Orr, 1979 \\
\hline Triangle double wing & latest Eocene-middle Miocene & Kozarek and Orr, 1979 \\
\hline cf. Five peaks flared base & early Eocene-early Oligocene & Doyle et al., 1974 \\
\hline Flexed triangle shallow inbase $\geq 120$ & early Oligocene-late Miocene & Doyle and Riedel, 1985a \\
\hline Narrow triangle straight inbase & early middle Eocene-Holocene & Kozarek and Orr, 1979 \\
\hline Kite shaped irregular network & early Eocene-Oligocene & Kozarek and Orr, 1979 \\
\hline Asymmetrical peak wide depression & early Oligocene-middle Miocene & Kozarek and Orr, 1979 \\
\hline Many peaks transverse lines & middle Eocene-Pliocene & Tway et al., 1985 \\
\hline Short kite shaped & middle Eocene-late Oligocene & Doyle et al., 1974 \\
\hline Tanged triangle & Paleocene-early Miocene & Gottfried et al., 1984a, b \\
\hline Five peaks flared base & early Eocene-middle Miocene & Kozarek and Orr, 1979 \\
\hline cf. Pointed triangle short inline & early Eocene-middle Miocene & Gottfried et al., 1984a \\
\hline \multirow[t]{2}{*}{ Kite shaped elongate prominence } & early Eocene-late Oligocene & Doyle et al., 1974; Kozarek and Orr, 1979 \\
\hline & ?Pale & Winfrey et al., 1987 \\
\hline Long triangle thin wall & early Eocene-late Oligocene & Kozarek and Orr, 1979 \\
\hline Triangle with base angle & late Eocene-Holocene & Doyle and Riedel, 1985a \\
\hline Rectangular irregularly sawtoothed & Paleocene?/Eocene-Holocene & Doyle et al., 1979a, Gottfried et al., 1984b. \\
\hline Large fibrous triangle & middle Paleocene-middle Miocene & Tway et al., 1985 \\
\hline Three equal peaks flared base & middle Eocene-early Oligocene & Kozarek and Orr, 1979 \\
\hline Elliptical with line across & middle Miocene-Holocene & Doyle and Riedel, $1985 \mathrm{a}$ \\
\hline Pointed triangle long margins & middle Eocene-Pliocene & Gottfried et al., 1984a; Doyle and Riedel, 1985b \\
\hline Small triangle crenate margin & early Eocene-early Oligocene & Doyle and Riedel, $1985 \mathrm{a}$ \\
\hline Small dendritic many radiating lines & early Oligocene-Holocene & Doyle and Riedel, 1985a \\
\hline Curved triangle pointed margin & middle Eocene-Holocene & Doyle and Riedel, 1985a \\
\hline Asymmetrical peaks narrow depression & early Eocene-middle Miocene & Doyle et al., 1974 \\
\hline Triangle crenulate & late Eocene-Pliocene & Kozarek and Orr, 1979 \\
\hline Stippled triangle & Oligocene/Miocene boundary-Holocene & Doyle and Riedel, $1985 \mathrm{a}$ \\
\hline Flexed triangle $102-112$ & late Oligocene-Pliocene & Doyle and Riedel, 1985a \\
\hline cf. Triangle inline halfway & early Eocene-middle Miocene & Kozarek and Orr, 1979 \\
\hline Triangle sigmoid & early Eocene-Pliocene & Kozarek and Orr, 1979 \\
\hline Skewed with transverse lines & Oligocene-Miocene & Winfrey et al., 1987 \\
\hline Small triangle long striations & Oligocene/Miocene boundary-Holocene & Doyle and Riedel, 1985a \\
\hline Triangle hooked margin & early Eocene-Holocene & Doyle and Riedel, 1985a \\
\hline Narrow triangle ragged base & early Miocene-Holocene & Doyle and Riedel, 1985a \\
\hline Short triangle stepped margin & Oligocene/Miocene boundary-Holocene & Doyle and Riedel, 1985a \\
\hline Triangle sinuous inline & Oligocene/Miocene boundary-Holocene & Gottfried et al., 1984a \\
\hline Triangle broad wing & middle Paleocene-middle Eocene & Doyle and Riedel, 1985a \\
\hline Triangle curved base & early Paleocene-early Eocene & Doyle and Riedel, 1985a \\
\hline Narrow triangle unequal margins & Paleocene & Doyle and Riedel, 1985b \\
\hline Triangle long inline & Cretaceous-early Paleocene & Doyle et al., 1978 \\
\hline Triangle bowed inline & Cretaceous & Ramsey et al., 1976 \\
\hline
\end{tabular}

sediments in the region. Three equal peaks flared base and Small triangle crenate margin have reported ranges of middle and upper Eocene to lower Oligocene, whereas Seven peaks and Long triangle thin wall have reported ranges into the upper Oligocene (Table 2). It is uncertain whether their occurrences in Hole 842B represent reworking or extensions of previously reported ranges.

The source and extent of exposed older sediments is unknown, but they indicate the occurrence of some form of lateral transport on the seafloor during the deposition of pelagic clays in the early Miocene. Early to middle Eocene radiolarians have been observed within ash layers in Core $842 \mathrm{~A}-1 \mathrm{H}$ (Hull, this volume). The sediments in Core 842A-1H are of Quaternary age (Dziewonski, Wilkens, Firth et al., 1992). Similar mixtures of Eocene and Quaternary radiolarians have been reported at DSDP Sites 67 (Winterer, Riedel, et al., 1971) and 68 (Tracey, Sutton, et al., 1972), near the Hawaiian Arch. Similar processes of reworking and deposition are suggested during both the early Miocene and Quaternary at Site 842.

\section{CONCLUSIONS}

Ichthyolith biostratigraphy of deep-sea red clays recovered at Site 842 on the southwest Hawaiian Arch indicates an age of early Miocene between 31.10 and 21.53 mbsf. Sediments below this interval, down to $35.57 \mathrm{mbsf}$, are of Oligocene to early Miocene age. The interval between 21.53 and 19.05 mbsf is of early to middle Miocene age. Sediments immediately overlying and underlying the studied intervals are barren of all microfossils, and their ages are unknown.

Reworked ichthyoliths are evident in the studied intervals and indicate sources of Cretaceous to Eocene age exposed on the seafloor during the early Miocene. 


\section{SYSTEMATIC TAXONOMY}

The system of nomenclature developed by Doyle et al. (1974), and subsequently modified by Dunsworth et al. (1975), Ramsey et al. (1976) (Mesozoic forms, presumably reworked in these samples), Doyle et al. (1978), Doyle and Riedel (1979b), Tway (1979), Gottfried et al. (1984a), Doyle and Riedel (1985b), Tway et al. (1985), and Winfrey et al. (1987), was followed in this paper. Some undescribed forms are described and discussed herein, but are not formally named.

Skewed 4 or 5 peaks Doyle et al., 1974 (Pl. 2, Fig. 1) $\mathrm{a} 2 / \mathrm{b} 2 / \mathrm{c} 3 / \mathrm{d} 2,3 / \mathrm{e} 1 / \mathrm{f} 1 / \mathrm{g} 1,2 / \mathrm{h} 4 / \mathrm{i} 1,2 / \mathrm{j} 1$

Skewed with transverse lines Doyle et al., 1974 (PI. 2, Fig. 7) $\mathrm{a} 2 / \mathrm{b} 2 / \mathrm{c} 3 / \mathrm{d} 1 / \mathrm{e} 2 / \mathrm{f} 2 / \mathrm{g} 1 / \mathrm{h} 1,2 / \mathrm{i} 1 / \mathrm{j} 2,(2+3)$

Three equal peaks flared base Doyle et al., 1974 (PI. 2, Fig. 6) $\mathrm{a} 2 / \mathrm{b} 2 / \mathrm{c} 3 / \mathrm{d} 2,3 / \mathrm{e} 1 / \mathrm{f1} / \mathrm{g} 1,2 / \mathrm{h} 4 / \mathrm{i} 1,2 / \mathrm{j} 1$

Five peaks flared base Doyle et al., 1974 (PI. 2, Fig. 3) $\mathrm{a} 2 / \mathrm{b} 2 / \mathrm{c} 5 / \mathrm{d} 3 / \mathrm{e} 1 / \mathrm{f1} / \mathrm{g} 1 / \mathrm{h} 4 / \mathrm{i} 1 / \mathrm{j} 1,2$

cf. Five peaks flared base Doyle et al., 1974 (PI. 2, Fig. 4) $\mathrm{a} 2 / \mathrm{b} 2 / \mathrm{c} 5 / \mathrm{d} 3 / \mathrm{e} 1 / \mathrm{f} 1 / \mathrm{g} 1 / \mathrm{h} 4 / \mathrm{i} 1 / \mathrm{j} 1,2$

Remarks: U-shaped depressions present between peaks on Five peaks flared base are shallow to absent on the form illustrated herein.

Many peaks transverse lines Tway et al., 1985 (PI. 2, Figs. 8, 11 ) $\mathrm{a} 2 / \mathrm{b} 2 / \mathrm{c5}, 6,7 / \mathrm{d} 0.89-1.09 / \mathrm{e} 1,2 / \mathrm{f} 3 / \mathrm{g} 1,2 / \mathrm{h} 1,3 / \mathrm{il} 1 / \mathrm{j} 2+3 / \mathrm{k} 2$

Seven peaks Kozarek and Orr, 1979 (Pl. 2, Figs. 9, 10) $\mathrm{a} 2 / \mathrm{b} 2 / \mathrm{c} 7 / \mathrm{d} 1 / \mathrm{e} 1 / \mathrm{f} 1 / \mathrm{g} 2 / \mathrm{h} 1 / \mathrm{i} 1 / \mathrm{j} 1 / \mathrm{k} 2$

Short kite-shaped Doyle et al., 1974 (PI. 1, Fig. 6) $\mathrm{a} 3 / \mathrm{b} 1 / \mathrm{c} 3 / \mathrm{d} 1,3 / \mathrm{e} 1 / \mathrm{f1},(2+3) / \mathrm{g} 1+2$

Remarks: This form appears to be equivalent to Lined lanceolate differ- entiated margin of Tway et al. (1985). Short kite-shaped is retained as the name of this form by priority.

Kite-shaped irregular network Doyle et al., 1974 (Pl. 1. Fig. 1) a3/b1/c3/d4/e2/f2/g1+4

Polygonal cavity long rays Kozarek and Orr. 1979 (PI. 1, Fig. 5) $\mathrm{a} 3 / \mathrm{b} 1 / \mathrm{c} 4 / \mathrm{d} 5 / \mathrm{e} 1 / \mathrm{f} 1 / \mathrm{g} 1+2$

Polygonal cavity Kozarek and Orr, 1979 (Pl. 1, Figs. 2-4) a3/b1/c5/d5/e1/f1/g1+2

Kite-shaped elongate prominence Doyle et al., 1974 (PI. 1, Fig. 8) $\mathrm{a} 3 / \mathrm{b} 1 / \mathrm{c} 3 / \mathrm{d} 3,4 / \mathrm{e} 2,3,4 / \mathrm{f2}+3 / \mathrm{g} 1+8$

Kite-shaped longitudinal line Doyle et al., 1974 (PI. 1, Fig. 7) $\mathrm{a} 3 / \mathrm{b} 1 / \mathrm{c} 3 / \mathrm{d} 2 / \mathrm{e} 2 / \mathrm{f} 2+3 / \mathrm{g} 1+2$

Plain and lined lanceolate Doyle et al., 1974 (emend. Winfrey et al., 1987) (PI. 2, Fig. 2)

$\mathrm{a} 4 / \mathrm{b} 1,2 / \mathrm{c} 2 / \mathrm{d} 1,4 / \mathrm{e} 2 / \mathrm{f} 2+3 / \mathrm{g} 1+2 / \mathrm{h} 1$

Ogee lanceolate Tway et al., 1985 (PI. 3, Figs. 1, 2) $\mathrm{a} 4 / \mathrm{b} 1 / \mathrm{c} 2 / \mathrm{d} 6 / \mathrm{e} 1,2 / \mathrm{f2}+3 / \mathrm{g} 1+2 / \mathrm{h} 3$

Small dendritic many radiating lines Doyle et al., 1974 (PI. 3, Fig. 6 ) a5,6/b3/c2/d2/e1, 2

Elliptical with line across Doyle et al., 1974 (PI. 3, Fig. 4) $\mathrm{a} 6 / \mathrm{b} 4 / \mathrm{c} 2$

Rectangular sawtoothed Doyle et al., 1974 (PI. 3, Fig. 10) a7/b1/c2/d3/e6

Rectangular irregularly sawtoothed Doyle and Riedel, 1979b (Pl. 3, Fig. 9) $\mathrm{a} 7 / \mathrm{b} 1 / \mathrm{c} 2 / \mathrm{d} 3 / \mathrm{e} 7,8,9$

Rectangular triangular toothed Kozarek and Orr, 1979 (Pl. 3, Fig. 5) a $7 / \mathrm{b} 1,5 / \mathrm{c} 1 / \mathrm{d} 3 / \mathrm{e} 5$

Two triangles Doyle et al., 1974 (Pl. 3, Fig. 4) a $7 / \mathrm{b} 1 / \mathrm{c} 1 / \mathrm{d} 4 / \mathrm{e} 4$

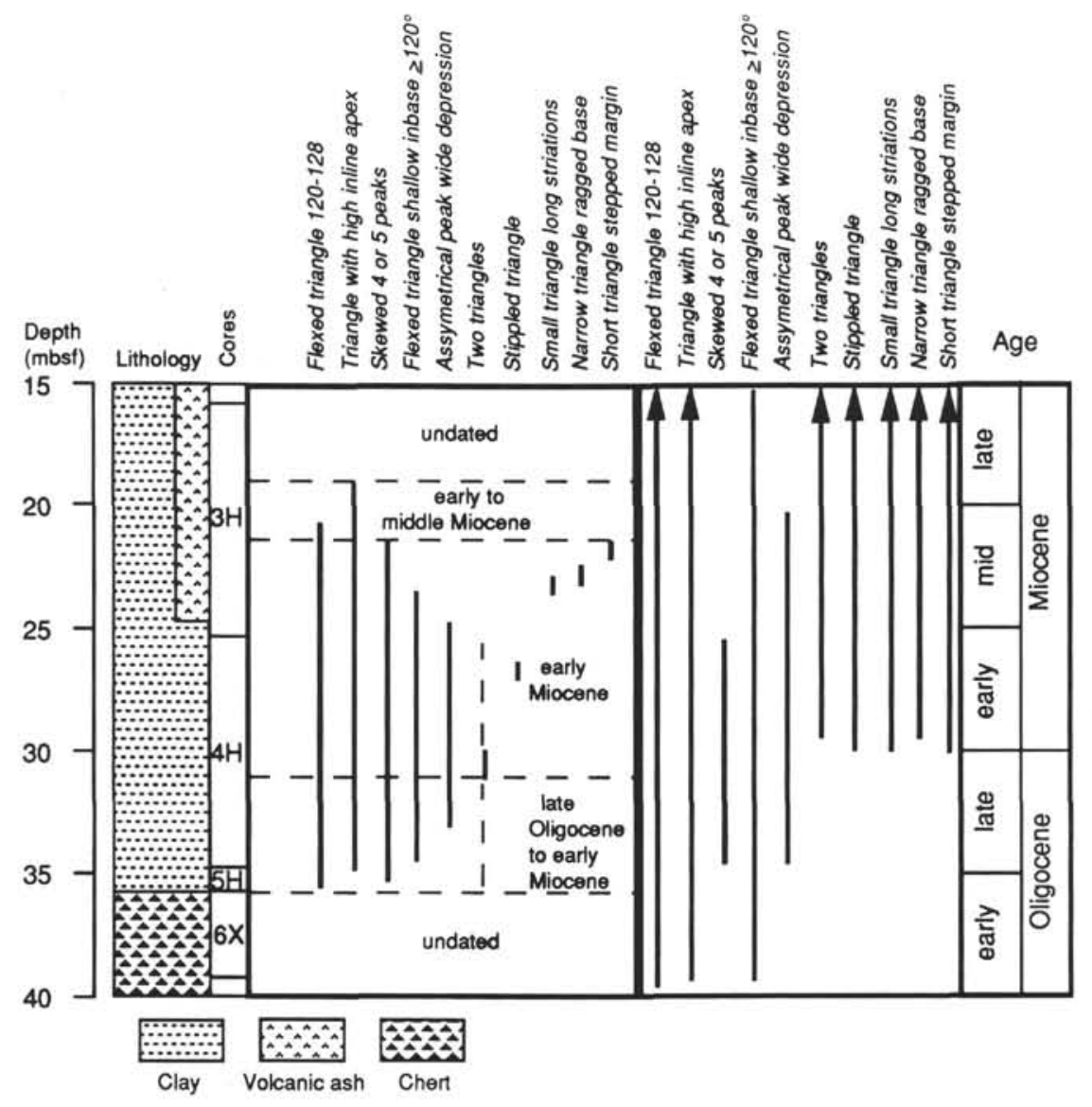

Figure 2. Ranges of selected ichthyolith taxa in Hole 842B on the left, and their corresponding reported age ranges in Doyle and Riedel (1985a) on the right (except for Skewed 4 or 5 peaks and Asymmetrical peak wide depression, the ranges of which are listed in Table 2). Dashed lines show range of forms comparable to (cf.) the indicated taxon. 
cf. Two triangles Doyle et al., 1974 (PI. 3, Fig. 8)

Remarks: This form is characterized by two triangles that are closely adjacent and appear to have a well-developed inline that follows the outline of the tooth.

Asymmetrical peak wide depression Doyle et al., 1974 (PI. 3, Fig. 7) a7/b6/c1

Asymmetrical peaks narrow depression Doyle et al., 1974 (PI. 3, Fig. 11) a7/b6/c3

Flexed triangle shallow inbase Doyle et al., 1974 (Pl. 4, Figs. 2, 3) $\mathrm{a} 8 / \mathrm{b} 1,5 / \mathrm{c} 1 / \mathrm{d} 2 / \mathrm{e} 80-140 / \mathrm{f} 26-36$

Flexed triangle shallow inbase $\geq 120$ Dunsworth et al., 1975 (PI. 4, Figs. 6,7) $\mathrm{a} 8 / \mathrm{b} 1,5 / \mathrm{c} 1 / \mathrm{d} 2,3 / \mathrm{e} \geq 120 / \mathrm{f} \leq 25+\mathrm{a} 9 / \mathrm{b} 1 / \mathrm{c} 13 / \mathrm{d} 1 / \mathrm{e} 1 / \mathrm{f} 4+(1,8) / \mathrm{g} 1 / \mathrm{h} 2,3,4,5 / \mathrm{i} 2 / \mathrm{j} 2 /$ $\mathrm{k} 4,8 / \mathrm{m} 0.25-0.45 / \mathrm{n} 2.00-3.00 / \mathrm{p} 0 / \mathrm{q} 2,3,4,6,7 / \mathrm{r} 2 / \mathrm{s} 1 / \mathrm{t} 1$

Flexed triangle 102-112 Doyle et al., 1974 (PI. 4. Fig. 4) $\mathrm{a} 8 / \mathrm{b} 1,5 / \mathrm{c1} / \mathrm{d} 1 / \mathrm{e} 102-112 / \mathrm{f} 26-36$

Flexed narrow triangle 120-128 Doyle et al., 1974 (PI. 4, Fig. 5) $\mathrm{a} 8 / \mathrm{b} 1,5 / \mathrm{c} 1 / \mathrm{d} 1 / \mathrm{e} 120-128 / \mathrm{f20}-26$

Triangle short wing Doyle et al., 1974 (PI. 5, Figs. 6,7) a9/b1/c5/d1/e1/f4+5/g1/h2,3,4,5/i2,3/j6/k8/m<0.4/n1.50-2.00/p2.00 $-4.00 / \mathrm{q} 1,9 / \mathrm{r} 1 / \mathrm{s} 1,2 / \mathrm{t} 1$

Triangle medium wing Doyle et al., 1974 (Pl. 5, Fig. 5) a9/b1/c6,7/d1/e1/f4+5/g1/h2,3,4,5/i2,3/j6/k8/m<0.40/n1.50-2.00/ p2.00-4.00/q1,9/r1/s1,2/t1

Triangle broad wing Doyle et al., 1974 (PI. 5, Fig. 8) $\mathrm{a} 9 / \mathrm{b} 1 / \mathrm{c} 6 / \mathrm{d} 1 / \mathrm{el} / \mathrm{f} 1,4 / \mathrm{g} 1 / \mathrm{h} 3,4,5 / \mathrm{i} 2 / \mathrm{j} 2,6 / \mathrm{k} 8 / \mathrm{m}>0.15 / \mathrm{n}<1.40 / \mathrm{p} 2.00-3.00 /$ $\mathrm{q} 9 / \mathrm{r} 1 / \mathrm{s} 1,2 / \mathrm{t} 1$

Triangle double wing Kozarek and Orr, 1979 (PI. 5, Fig. 4) a9/b1/c6/d6,7/e1/f1/g1/h5/i2,6/j2/k8/10.2-0.5/m2.2-3.0/n2/01/p2,3

Triangle pointed margin ends Doyle et al., 1974 (PI. 6, Fig. 2) a $9 / \mathrm{b} 1,5 / \mathrm{c} 13 / \mathrm{d} 13 / \mathrm{e} 1 / \mathrm{f} 4 / \mathrm{g} 1 / \mathrm{h} 0,5 / \mathrm{i} 2 / \mathrm{j} 2 / \mathrm{k} 3,8 / \mathrm{m}<0.60 / \mathrm{n} 2.00-3.00 / \mathrm{p} 2.0$ $3.00 / \mathrm{q} 0,3 / \mathrm{r} 0,3 / \mathrm{s} 1,2 / \mathrm{t} 4 / \mathrm{z} 11 / \mathrm{cc} 1 / \mathrm{dd} 1 / \mathrm{ee} 1 / \mathrm{ff} 1 / \mathrm{gg} 1 / \mathrm{hh} 0.50-3.00 / \mathrm{jj} 2 / \mathrm{kk} 2 /$ $\mathrm{mm} 0 / \mathrm{nn} 0$

cf. Triangle pointed margin ends Doyle et al., 1974 (Pl. 6, Figs. 1, 3) Remarks: Forms described as cf. Triangle pointed margin ends include specimens that have less angular margins at the base, length to width ratios greater than 2.0-3.0, and inlines that are at the same level or slightly below the margin ends at the base of the specimen.

Triangle transverse line across Doyle et al., 1974 (PI. 7. Fig. 3) $\mathrm{a} 9 / \mathrm{b} 1,5 / \mathrm{c} 1 / \mathrm{d} 1 / \mathrm{e} 1,2 / \mathrm{f} 4 / \mathrm{g} 1 / \mathrm{h} 0,5 / \mathrm{i} 4 / \mathrm{j} 4 / \mathrm{k} 3,8 / \mathrm{m} 0.20-0.40 / \mathrm{n} 1.50-2.50 /$ p1.50-2.60/q6,7,8/r4/s1/t1/z4/cc1/dd1/ee2/ff1/gg1/hh0/jj1,2,3/kk1, 2,3/mm0/nn0

Triangle with canals Doyle et al., 1974 (PI. 7, Figs, 1, 5) $\mathrm{a} 9 / \mathrm{b} 5 / \mathrm{c} 1 / \mathrm{d} 1 / \mathrm{e} 1 / \mathrm{f} 1 / \mathrm{g} 1,2 / \mathrm{h} 2 / \mathrm{i} 1,4 / \mathrm{j} 1 / \mathrm{k} 2 / 12 / \mathrm{m} 2 / \mathrm{n} 2 / 05,6 / \mathrm{p} 3 / \mathrm{q} 0.2-0.5 / \mathrm{r} 1-$ $1.5 / \mathrm{s} 0 / \mathrm{t} 1$

Triangle with triangular projection Doyle et al.. 1974 (Pl. 7. Fig. 4) a9/b5/c1/d1/e1/f1,8/g1/h0/i2,7/j2,3/k0,5,7/m0/n>1.00/p0/q0/r0/s2/t1/ $\mathrm{z} 2 / \mathrm{cc} 3 / \mathrm{dd} 1 / \mathrm{ee} 1 / \mathrm{ff} 1 / \mathrm{gg} 1 / \mathrm{hh}>3.00 / \mathrm{jj} 2,4 / \mathrm{kk} 3 / \mathrm{mm} 1.40-4.50 / \mathrm{nn} 0$

Narrow triangle cross-hachured Doyle et al., 1974 (PI. 7, Fig. 7) a9/b5/c1,4/d1/e1/f1,16,18/g1/h0/i2,7/j2/k0,5,8/m0/n>0.50/p0/q0/r0/s2/ t1/z2/cc1,3/dd1/ee1/ff1/gg2/hh>3.00/ji2,4/kk2,3/mm1.70-4.50/nn0

Short triangle stepped margin Doyle et al., 1974 (Pl. 8, Fig. 5) a $9 / \mathrm{b} 5 / \mathrm{c} 1 / \mathrm{d} 1 / \mathrm{e} 1 / \mathrm{f}(1,3)+(1,16,18) / \mathrm{g} 1 / \mathrm{h} 0 / \mathrm{i} 2,6 / \mathrm{j} 3 / \mathrm{k} 0,7,8 / \mathrm{m}>0.25 / \mathrm{n}<2.00 /$ $\mathrm{p} 0 / \mathrm{q} 0 / \mathrm{r} 0 / \mathrm{s} 2 / \mathrm{t} 1 / \mathrm{z} 3 / \mathrm{cc} 1 / \mathrm{dd} 1 / \mathrm{ee} 1 / \mathrm{ff} 1 / \mathrm{gg} 1 / \mathrm{hh}>1.00 / \mathrm{jj} 2,4 / \mathrm{kk} 3 / \mathrm{mm} 1.20$ $1.60 / \mathrm{nn} 0$

Triangle with high inline apex Doyle et al., 1974 (PI. 7, Fig. 2) $\mathrm{a} 9 / \mathrm{b} 1 / \mathrm{cl} / \mathrm{d} 1 / \mathrm{e} 1 / \mathrm{f} 1,4,8,21 / \mathrm{g} 1 / \mathrm{h} 2,3,4,5 / \mathrm{i} 2,6,8 / \mathrm{j} 2,3 / \mathrm{k} 3,8 / \mathrm{m}<0.20 / \mathrm{n} 1.50$ $2.00 / \mathrm{p} 1.50-2.00 / \mathrm{q} 3,4,5 / \mathrm{r} 1 / \mathrm{s} 1 / \mathrm{t} 1$

Narrow triangle straight inbase Doyle et al., 1974 (PI. 9, Fig. 1) $\mathrm{a} 9 / \mathrm{b} 1 / \mathrm{c} 1 / \mathrm{d} 1 / \mathrm{e} 1,2 / \mathrm{f} 1 / \mathrm{g} 1 / \mathrm{h} 1,2 / \mathrm{i} 2 / \mathrm{j} 2 / \mathrm{k} 2 / 10.2-0.4 / \mathrm{m} 1.6-2.0 / \mathrm{n} 4,5 / \mathrm{o} 1 / \mathrm{p} 1,3$

Wide triangle straight inbase Doyle et al., 1974 (PI. 9. Fig. 3) $\mathrm{a} 9 / \mathrm{b} 1 / \mathrm{c} 1 / \mathrm{d} 1 / \mathrm{e} 1 / \mathrm{fl} / \mathrm{g} 1 / \mathrm{h} 1,2,3 / \mathrm{i} 2,3 / \mathrm{j} 2,3 / \mathrm{k} 2 / 10.25-0.45 / \mathrm{ml}-1.5 / \mathrm{n} 4,5 / \mathrm{ol} / \mathrm{p} 3$

Pointed triangle long margins Gottfried et al., 1984a (PI. 9, Figs. 4, 5) a9/b1/c1/d1/e1/f4+17/g1/h2,5/i2,4/j2,4/k5,8/m0.45-0.80/n1.15-1.50/ p1.10-1.85/q1,8,9/r1/s2/t1

Tanged triangle Gottfried et al., 1984a (PI. 5, Fig. 1) $\mathrm{a} 9 / \mathrm{b} 1 / \mathrm{c} 1 / \mathrm{d} 1 / \mathrm{e} 1 / \mathrm{f} 4 / \mathrm{g} 1 / \mathrm{h} 0 / \mathrm{i} 2 / \mathrm{j} 2 / \mathrm{k} 0,4,7 / \mathrm{m} 0.55-1.00 / \mathrm{n} 1.75-1.95 / \mathrm{p} 0 / \mathrm{q} 10 /$ $\mathrm{r} 1 / \mathrm{s} 1 / \mathrm{t} 1$

Triangle concave base Doyle et al., 1978 (Pl. 10. Figs. 2, 3) $\mathrm{a} 9 / \mathrm{b} 1 / \mathrm{c} 1 / \mathrm{d} 1 / \mathrm{e} 1 / \mathrm{f} 1 / \mathrm{g} 1 / \mathrm{h} 2,4,5 / \mathrm{i} 2,6 / \mathrm{j} 2 / \mathrm{k} 8 / 10.2-0.3 / \mathrm{m} 1.9-2.5 / \mathrm{n} 6,7,8 / \mathrm{o} 1,3 /$ p1,2/q1

cf. Pointed triangle short inline Gottfried et al., 1984a (PI. 6, Fig. 7) $\mathrm{a} 9 / \mathrm{b} 1 / \mathrm{c} 1 / \mathrm{d} 1 / \mathrm{e} 1 / \mathrm{f}(1,14)+4 / \mathrm{g} 1 / \mathrm{h} 5 / \mathrm{i} 2 / \mathrm{j} 2 / \mathrm{k} 5,7 / \mathrm{m} 0.70-0.90 / \mathrm{n} 2.10-3.20 /$ $\mathrm{p} 0 / \mathrm{q} 2,6,7 / \mathrm{r} 1,5 / \mathrm{s} 2 / \mathrm{t} 1$
Stippled triangle Dunsworth et al., 1975 (Pl. 10, Fig. 4) a $9 / \mathrm{b} 5 / \mathrm{c} 1 / \mathrm{d} 1 / \mathrm{e} 1 / \mathrm{f} 1 / \mathrm{g} 1 / \mathrm{h} 0 / \mathrm{i} 2 / \mathrm{j} 2 / \mathrm{k} 0 / \mathrm{m} 0 / \mathrm{n} 0 / \mathrm{p} 0 / \mathrm{q} 0 / \mathrm{r} 0 / \mathrm{s} 1,2 / \mathrm{t} 1 / \mathrm{z} 2,3 / \mathrm{cc} 2 / \mathrm{dd} 1$, 2/ee2/ff1/gg4/hh>2.00/jj4,6/kk1,3/mm0/nn0

Triangle curved base Doyle et al., 1978 (Pl. 5, Fig. 3) $\mathrm{a} 9 / \mathrm{b} 1,5 / \mathrm{c} 1 / \mathrm{d} 1 / \mathrm{e} 1,2 / \mathrm{f} 4 / \mathrm{g} 1 / \mathrm{h} 0,5 / \mathrm{i} 2,3 / \mathrm{j} 2,3 / \mathrm{k} 3,7 / \mathrm{m} 0.50-0.70 / \mathrm{n} 1.50-2.30 /$ p1.50—2.50/q0,6,7/r0,1,3,4/s1/t1/z4/cc1/dd1/ee2/ff1/gg1/hh0/jj1,2/ kk1,2/mm1.00-1.15/nn0

Triangle with base angle Dunsworth et al., 1975 (Pl. 4, Fig. 1) $\mathrm{a} 9 / \mathrm{b} 1,5 / \mathrm{c} 9,13 / \mathrm{d} 1 / \mathrm{el} / \mathrm{f} 4+8 / \mathrm{g} 1 / \mathrm{h} 0,4 / \mathrm{i} 2 / \mathrm{j} 2 / \mathrm{k} 7,8 / \mathrm{m} 0.20-0.60 / \mathrm{n} 1.60-2.80 /$ p0/q0,2/r0,1,2/s1/tt/z9/cc1/dd1/ee1/ff1/gg1/hh0/jj2/kk2/mm0/nn0

Large fibrous triangle Tway et al., 1985 (Pl. 6, Figs. 8, 9) $\mathrm{a} 9 / \mathrm{b} 1 / \mathrm{c} 1 / \mathrm{d} 1 / \mathrm{e} 1 / \mathrm{f} 1 / \mathrm{g} 4 / \mathrm{h} 2,3,4,5 / \mathrm{i} 2,7,8 / \mathrm{j} 2 / \mathrm{k} 4,9 / \mathrm{m} 0.23-0.5 / \mathrm{n} 1.67-2.36 /$ p1.67-2.36/q9,10/r1/s2/t1/z0

Narrow triangle unequal margins Doyle and Riedel, 1985b (PI. 9, Fig. 6) a9/b1,5/c13/d1,13/e1/f1/g1/h0,3,4/i2,3/j6/k8/m0.1-0.4/n1.8-2.5/p2.2 $-2.6 / \mathrm{q} 0,6 / \mathrm{r} 0,1,2,3 / \mathrm{s} 1,2 / \mathrm{t1} / \mathrm{z} 9 / \mathrm{cc} 0 / \mathrm{dd} 0 / \mathrm{ee} 0 / \mathrm{ff} 0 / \mathrm{gg} 0 / \mathrm{hh} 0 / \mathrm{jj} 0 / \mathrm{kk} 0 / \mathrm{mm} 0 /$ nno

Long triangle thin wall Dunsworth et al., 1975 (Pl. 10, Fig. 7) a9/b5/c1/d1/e1/f1/g1/h1/i1/j1/k2,6/12,3/m2,4/n2,3/o2/p2,3,7/q0/r1.5$2.5 / \mathrm{s} \geq 4.0 / \mathrm{t} 2 / \mathrm{u} 3$

Curved triangle pointed margin Doyle et al., 1974 (PI. 9, Fig. 2) $\mathrm{a} 9 / \mathrm{b} 1 / \mathrm{c1} / \mathrm{d} 1 / \mathrm{e} 1 / \mathrm{f2} / \mathrm{g} 1 / \mathrm{h} 1,2,3 / \mathrm{i} 6 / \mathrm{j} 4 / \mathrm{k} 2,4 / 10.2-0.4 / \mathrm{ml}-2 / \mathrm{n} 3,4,5 / \mathrm{o} 2 / \mathrm{p} 1$

Curved triangle pointed inline Tway et al., 1985 (PI. 9, Fig. 7) $\mathrm{a} 9 / \mathrm{b} 1 / \mathrm{c} 1 / \mathrm{d} 1 / \mathrm{el} / \mathrm{f} 4 / \mathrm{g} 1 / \mathrm{h} 3,4,5 / \mathrm{i} 6 / \mathrm{j} 2,3 / \mathrm{k} 3,4 / \mathrm{m} 0.30-0.63 / \mathrm{n} 0.93-1.46 /$ p0.90-1.46/q6/r1,3/s2/t4/z0

Small triangle crenate margin Dunsworth et al., 1975 (PI. 5, Fig. 2) a9/b1,5/c15,17/el, 2/f1,17/g1/h0,5/i2,3/j2,3/k7,9/m0.25-0.50/n0.50 $1.50 / \mathrm{p} 0.70$ - $1.60 / \mathrm{q} 0,6,7,9 / \mathrm{r} 0,1 / \mathrm{s} 1,2 / \mathrm{t} 1 / \mathrm{z} 4 / \mathrm{ccl} / \mathrm{dd} 1 / \mathrm{ee} 1,2 / \mathrm{ffl} 1 / \mathrm{gg} 1 / \mathrm{hh} 1.00$ $-2.75 / \mathrm{jj} 1,2,3 / \mathrm{kk} 1,2,3 / \mathrm{mm} 0 / \mathrm{nn} 0$

Triangle crenulate Doyle et al., 1974 (PI. 8, Fig. 4) $\mathrm{a} 9 / \mathrm{b} 1 / \mathrm{c} 3 / \mathrm{d} 1,3 / \mathrm{el}, 2 / \mathrm{f} 1,2 / \mathrm{g} 1 / \mathrm{h} 1,2,3 / \mathrm{i} 2,6 / \mathrm{j} 2,3,6 / \mathrm{k} 2 / \mathrm{l}<0.3 / \mathrm{m} 1-2 / \mathrm{n} 4,5 / \mathrm{o} 1 /$ $\mathrm{p} 1,3$

cf. Triangle inline halfway Doyle et al., 1974 (PI. 7, Fig. 8) $\mathrm{a} 9 / \mathrm{b} 1 / \mathrm{c} 1 / \mathrm{d} 1 / \mathrm{e} 1 / \mathrm{f} 4 / \mathrm{g} 1 / \mathrm{h} 1 / \mathrm{i} 2 / \mathrm{j} 2 / \mathrm{k} 2 / 10.45-0.55 / \mathrm{m} 2.5-3.5 / \mathrm{n} 2 / 01,5 / \mathrm{p} 2$

Triangle sigmoid Dunsworth et al., 1975 (PI. 10, Fig. 6) a9/b1/c1/d1/e1/f1,3/g1/h5/i9/j9/k8/10.75-0.95/m $>2.75 / \mathrm{n} 2 / \mathrm{o} 1 / \mathrm{p} 2+\mathrm{a} 9 / \mathrm{b} 5 /$ $\mathrm{cl} / \mathrm{d} 1 / \mathrm{e} 1 / \mathrm{fl} / \mathrm{g} 1 / \mathrm{h} 1 / \mathrm{i} 1 / \mathrm{j} 1 / \mathrm{k} 9 / 19 / \mathrm{m} 1,4 / \mathrm{n} 1,3 / \mathrm{o} 9 / \mathrm{p} 3 / \mathrm{q} 0.75-0.95 / \mathrm{r} \geq 2.75 / \mathrm{s} 0 /$ t2/u1

Triangle inward angle Gottfried et al., 1984a (PI. 6, Fig. 5) $\mathrm{a} / \mathrm{b} 1 / \mathrm{c} 20 / \mathrm{d} 1 / \mathrm{e} 1 / \mathrm{f} 1,4 / \mathrm{g} 1 / \mathrm{h} 4 / \mathrm{i} 6 / \mathrm{j} 2 / \mathrm{k} 3,12+14 / \mathrm{m} 0.15-0.30 / \mathrm{n} 2.25-2.50 /$ p0/q3,6,9,10/r1/s1,2/t1

Triangle long inline Doyle et al., 1978 (PI. 10, Fig. 8) $\mathrm{a} 9 / \mathrm{b} 1 / \mathrm{cl} / \mathrm{d} 1 / \mathrm{e} 1 / \mathrm{f} 1,4 / \mathrm{g} 1 / \mathrm{h} 5 / \mathrm{i} 2,6 / \mathrm{j} 2,3 / \mathrm{k} 7,12 / \mathrm{m0} .05-0.40 / \mathrm{n} 2.50-4.99 / \mathrm{p} 0 /$ $\mathrm{q} 2 / \mathrm{r} 1 / \mathrm{s} 1,2 / \mathrm{t} 4$

Small triangle long striations Dunsworth et al., 1975 (PI. 8, Fig. 7) $\mathrm{a} 9 / \mathrm{b} 1,5 / \mathrm{c} 1,13 / \mathrm{d} 1 / \mathrm{e} 1 / \mathrm{f}(9,10)+13+14+18 / \mathrm{g} 1 / \mathrm{h} 0,5 / \mathrm{i} 2 / \mathrm{j} 2 / \mathrm{k} 0,3,5 / \mathrm{m} 0.75$ 1.00/n1.30-2.00/p0/q2/r1/s1,3/t1/z2/cc1/dd1/ee1/ff1/gg1/hh0/ $\mathrm{jj} 4 / \mathrm{kk} 3 / \mathrm{mm} 0 / \mathrm{nn} 0$

Triangle hooked margin Doyle et al., 1974 (PI. 10, Fig. 5) a $9 / \mathrm{b} 1,5 / \mathrm{c} 14 / \mathrm{d} 1 / \mathrm{el} / \mathrm{f5}+8 / \mathrm{g} 1 / \mathrm{h} 0,2 / \mathrm{i} 4,10 / \mathrm{j} 2,3 / \mathrm{k} 8 / \mathrm{m} 0.10-0.40 / \mathrm{n} 1.00-2.00 /$ p0/q0,1/r0,1/s1,2/t1/z10/cc1/dd1/ee1/ff1/gg1/hh0/jj2/kk3/mm0/nn0

Narrow triangle ragged base Dunsworth et al., 1975 (PI. 7, Fig. 6) a9/b1/c1/d1/e1/f1/g1/h3,4,5/i6/j3,5/k9/m0.20-0.70/n $\geq 4.00 / \mathrm{p} \geq 4.00 / \mathrm{q} 9 /$ $\mathrm{r} 1 / \mathrm{s} 1,3 / \mathrm{t} 1$

Triangle sinuous line Gottfried et al., 1984a (Pl. 10, Fig. 1) $\mathrm{a} 9 / \mathrm{b} 1 / \mathrm{c} 1 / \mathrm{d} 1 / \mathrm{e} 1 / \mathrm{f}(1,4)+21 / \mathrm{g} 1 / \mathrm{h} 0 / \mathrm{i} 2 / \mathrm{j} 2 / \mathrm{k}(4,8)+13 / \mathrm{m} 0.10-0.20 / \mathrm{n} 1.25-$ $1.60 / \mathrm{p} 0 / \mathrm{q} 4,9 / \mathrm{r} 1 / \mathrm{s} 1 / \mathrm{t} 1$

Triangle bowed inline Ramsay et al., 1976 (Pl. 8, Figs. 8, 9) $\mathrm{a} 9 / \mathrm{b} 1 / \mathrm{cl} / \mathrm{d} 1 / \mathrm{el} / \mathrm{f} 1,4 / \mathrm{g} 1 / \mathrm{h} 5 / \mathrm{i} 2 / \mathrm{j} 2 / \mathrm{k} 3,9 / 10.4-0.6 / \mathrm{m} 1.4-2.8 / \mathrm{u} 2,6 / \mathrm{o} 1 / \mathrm{p} 1,3 /$ q4

cf. Triangle bowed inline Ramsay et al., 1976 (Pl. 8, Fig. 10) Remarks: The inline of the specimen illustrated herein is of greater length and possesses a base that extends below the margin ends of the tooth.

Unnamed form A (PI. 8, Figs. 1-3) Remarks: Undescribed form of a9/bl,5.

Unnamed form B (PI. 2, Fig. 5) $\mathrm{a} 4 / \mathrm{b} 2 / \mathrm{c} 2 / \mathrm{d} 4 / \mathrm{e} 1 / \mathrm{f5} / \mathrm{g} 3 / \mathrm{h} 1$

Remarks: System of nomenclature modified by Tway (1979) is utilized to describe this form, which is characterized by the presence of a small platform at the base of the blade. This form differs from Three equal peaks flared base by having no depressions between the peaks.

Unnamed form C (Pl. 6, Fig. 4)

Remarks: Undescribed form of a9/bl,5. 
Unnamed form D (PI. 8, Fig. 6 )

Remarks: Undescribed form of a9/b1,5.

Unnamed form E (Pl. 6, Fig. 6)

Remarks: Undescribed form of a9/bl,5.

\section{ACKNOWLEDGMENTS}

This research was funded by a grant from USSAC. Reviews by Linda Tway and William Orr are much appreciated and helped improve this paper.

\section{REFERENCES}

Doyle, P.S., Dunsworth, M.J., and Riedel, W.R., 1978. Ichthyoliths from some southeast Atlantic sediments, DSDP Leg 40. In Bolli, H.M., Ryan, W.B.F., et al., Init. Repts. DSDP, 40 (Suppl., Pt. 4): Washington (U.S. Govt. Printing Office), 743-759.

Doyle, P.S., Kennedy, G.G., and Riedel, W.R., 1974. Stratignathy. In Davies, T.A., Luyendyk, B.P., et al., Init. Repts. DSDP. 26: Washington (U.S. Govt. Printing Office), 825-905.

Doyle, P.S., and Riedel, W.R., 1979a. Cretaceous to Neogene ichthyoliths in a giant piston core from the central North Pacific. Micropaleontology; 25:337-364.

1979b. Ichthyoliths: Present Status of Taxonomy and Stratigraphy of Microscopic Fish Skeletal Debris. Scripps Inst. Oceanogr. Ref. Ser., 79-16.

1985a. Cenozoic and Late Cretaceous ichthyoliths. In Bolli, H.M., Saunders, J.B., and Perch-Nielsen, K. (Eds.), Plankton Stratigraphy: Cambridge (Cambridge Univ. Press), 965-995.

1985b. Ichthyolith biostratigraphy of western North Pacific pelagic clays, Deep Sea Drilling Project Leg 86. In Heath, G.R., Burckle, L.H., et

\footnotetext{
Abbreviation for names of organizations and publications in ODP reference lists follow the style given in Chemical Abstracts Service Source Index (published by American Chemical Society).
}

al., Init. Repts. DSDP, 86: Washington (U.S. Govt. Printing Office), 349-366.

Dunsworth, M.J., Doyle, P.S., and Riedel, W.R., 1975. In Larson, R.L.. Moberly, R., et al., Init. Repts. DSDP, 32: Washington (U.S. Govt. Printing Office), 853-863.

Dziewonski, A., Wilkens, R., Firth, J., et al., 1992. Proc, ODP, Init. Repts., 136: College Station, TX (Ocean Drilling Program).

Gottfried, M.D., Doyle, P.S., and Riedel, W.R., 1984a. Advances in ichthyolith stratigraphy of the Pacific Neogene and Oligocene. Micropaleontology, 30:71-85.

, 1984b. Stratigraphic interpretations of pelagic sequences revised on the basis of ichthyoliths. Micropaleontology, 30:426-444.

Kozarek, R.J., and Orr, W.N., 1979. Ichthyoliths, Deep Sea Drilling Project Legs 51 through 53. In Donnelly, T., Francheteau, J., Bryan, W., Robinson, P., Flower, M., Salisbury, M., et al., Init. Repts. DSDP, 51, 52, 53 (Pt. 2): Washington (U.S. Govt. Printing Office), 857-895.

Ramsay, C.A., Doyle, P.S., and Riedel, W.R., 1976. Ichthyoliths in Late Mesozoic pelagic sediments, mainly from Italy. Micropaleontology, 22:129-142.

Tracey, J.I., Jr., Sutton, G.H., et al., 1971. Init. Repts, DSDP, 8: Washington (U.S. Govt. Printing Office).

Tway, L.E., 1979. A coded system for utilizing ichthyoliths of any age. Micropaleontology, 25:151-159.

Tway, L.E., Doyle, P.S., and Riedel, W.R., 1985. Correlation of dated and undated Pacific samples based on ichthyoliths and clustering techniques. Micropaleontology, 31:295-319.

Winfrey, E.C., Doyle, P.S., and Riedel, W.R., 1987. Preliminary ichthyolith biostratigraphy, southwest Pacific, Deep Sea Drilling Project Leg 91. In Menard, H.W., Natland, J., Jordan, T.H., Orcutt, J.A., et al., Init. Repts. DSDP, 91: Washington (U.S. Govt. Printing Office), 447-456.

Winterer, E.L., Riedel, W.R., et al., 1971. Init. Repts. DSDP, 7 (Pt. 1): Washington (U.S. Govt. Printing Office).

Date of initial receipt: 24 August 1992

Date of acceptance: 15 February 1993

Ms 136SR-202 


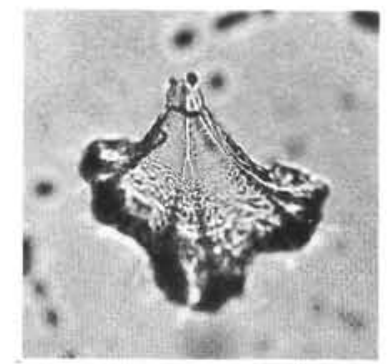

1

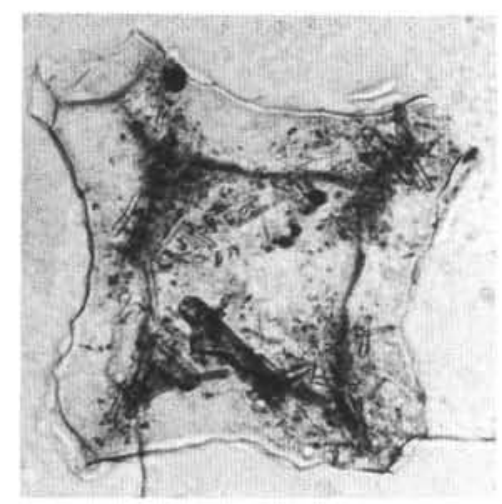

4

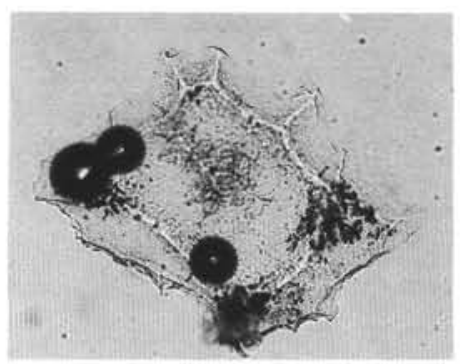

2

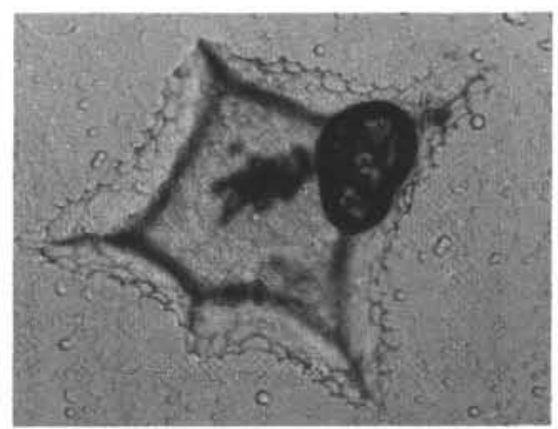

3

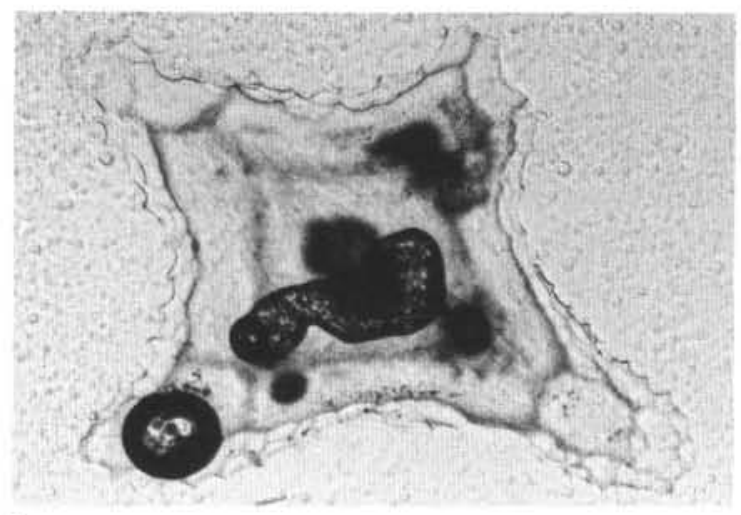

5

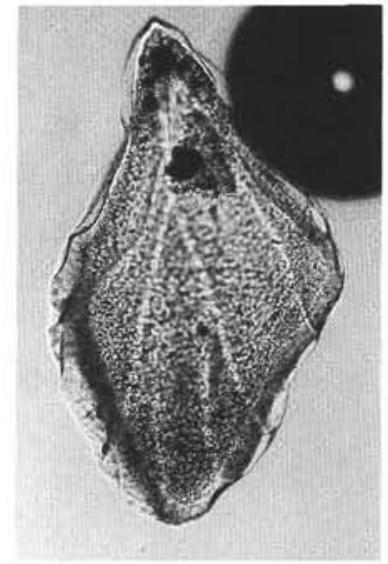

6
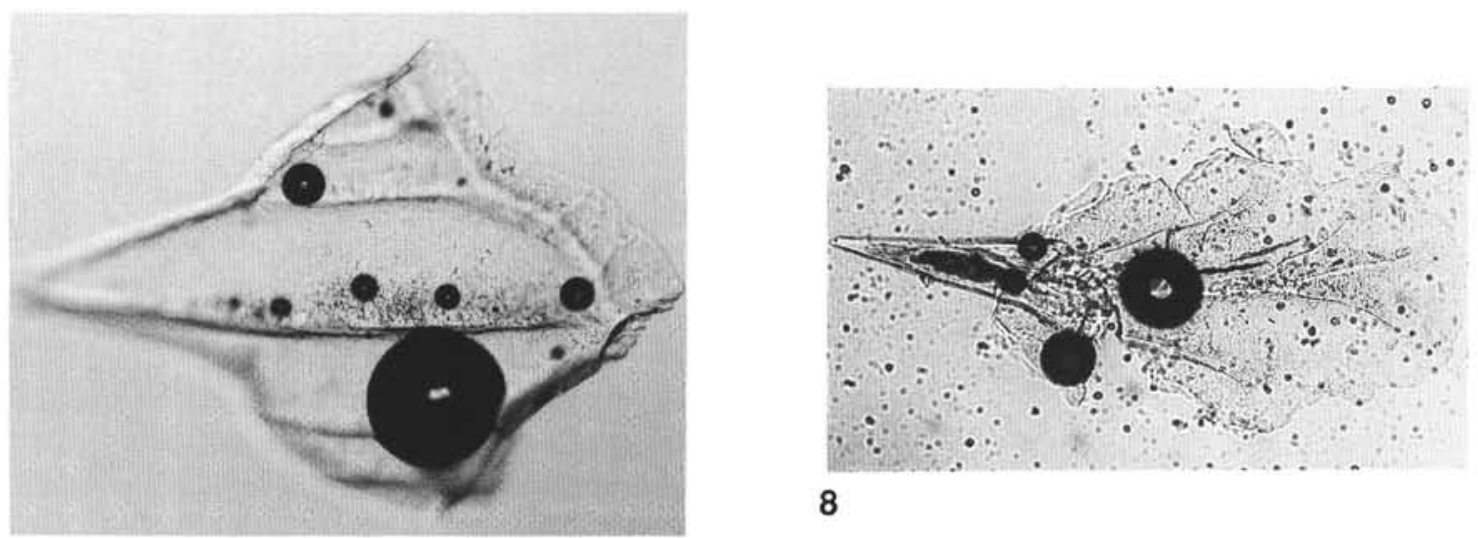

7

Plate 1. 1. Kite-shaped irregular network, Sample 842B-4H-6, 19-21 cm, 105×. 2,3,4. Polygonal cavity, Sample 842B-4H-5, 129-131 cm, 105×; Sample 842B-3H-4, 122-124 cm, 216x; Sample 842B-3H-7, 4-6 cm, 210x. 5. Polygonal cavity long rays, Sample 842B-3H-6, 27-29 cm, 202×. 6. Short kite-shaped, Sample 842B-4H-6, 19-21 cm, 105×. 7. Kite-shaped longitudinal line, Sample 842B-5H-CC, 105×. 8. Kite-shaped elongate prominence, Sample 842B-4H-4, 129-131 cm, 105x. 

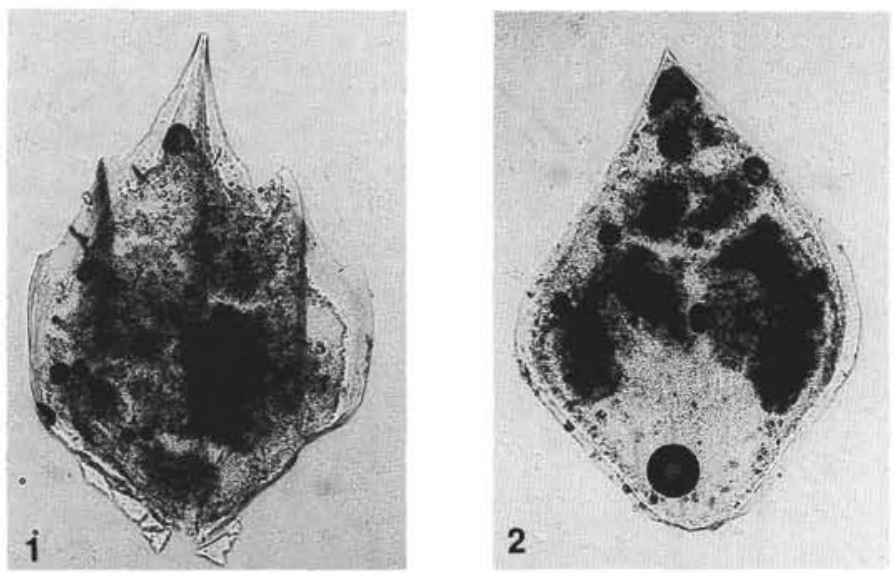
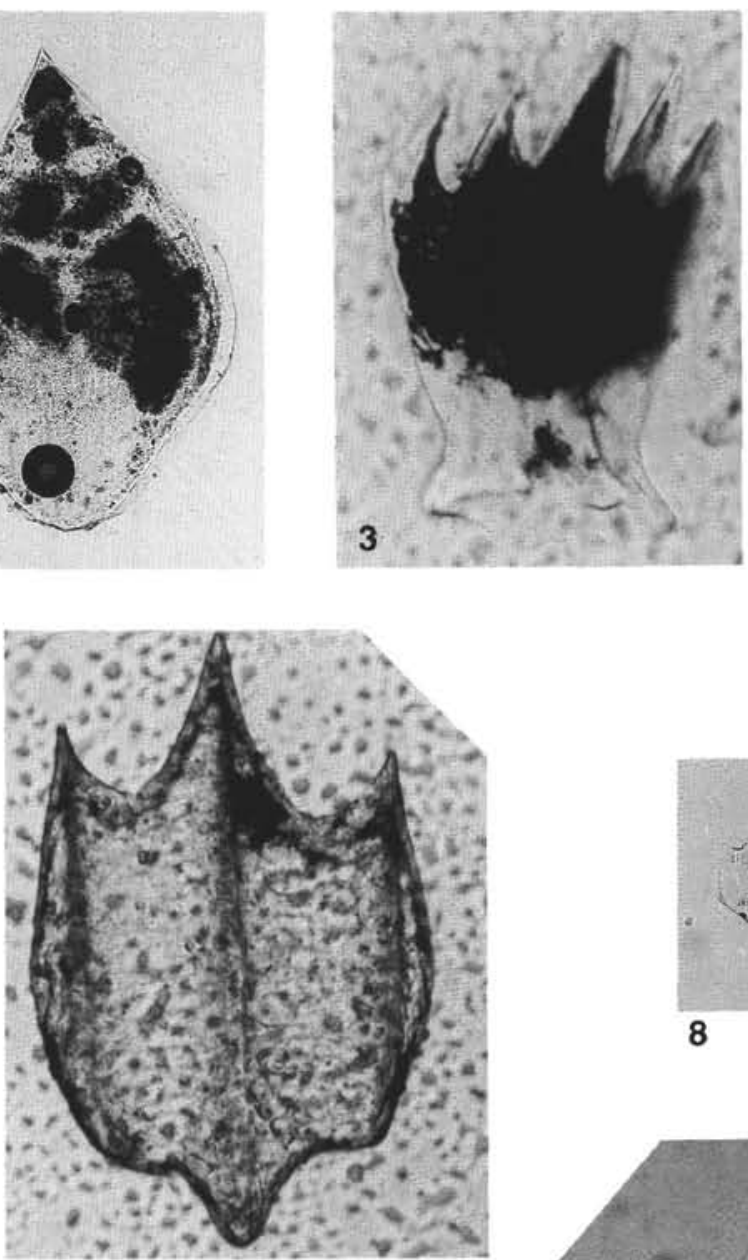

7

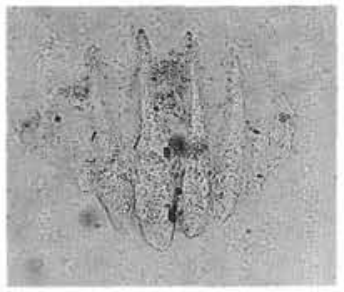

10

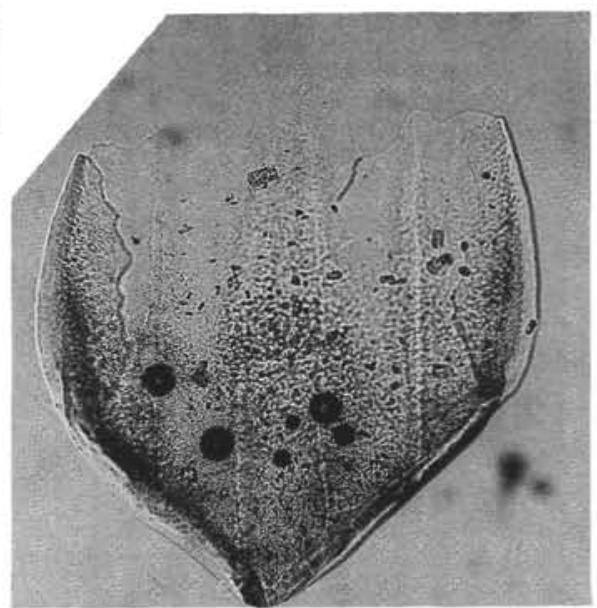

11

9

Plate 2. 1. Skewed 4 or 5 peaks, Sample $842 \mathrm{~B}-4 \mathrm{H}-\mathrm{CC}, 105 \times$. 2. Plain and lined lanceolate, Sample $842 \mathrm{~B}-4 \mathrm{H}-\mathrm{CC}$, 105×. 3. Five peaks flared base, Sample 842B-3H-6, 27-29 cm, 192×. 4. cf. Five peaks flared base, Sample 842B-4H-7, 20-22 cm, 105×. 5. Unnamed form B, Sample 842B-4H-7, 20-22 $\mathrm{cm}, 105 \times$. 6. Three equal peaks flared base, Sample 842B-4H-3, 130-132 cm, 105×. 7. Skewed with transverse lines, Sample 842B-3H-6, 124-126 cm, 207×. 8, 11. Many peaks transverse lines, Sample 842B-4H-3, 19-21 cm, 105×; Sample 842B-4H-6, 19-21 cm, 105×. 9, 10. Seven peaks, Sample 842B-4H-2, 19-21 cm, 104x; Sample 842B-4H-CC, 105x. 


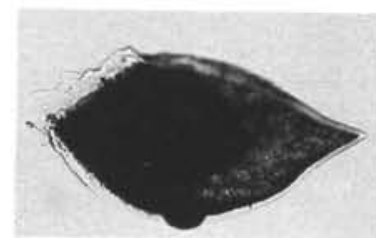

1

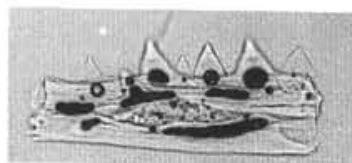

5

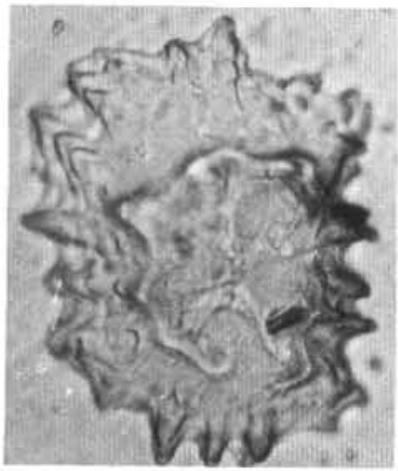

6

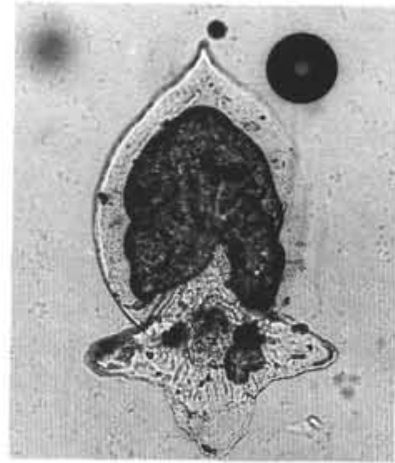

2

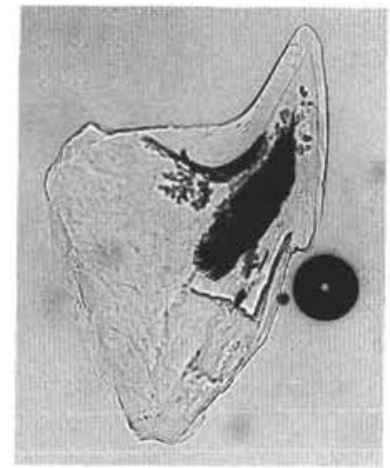

7

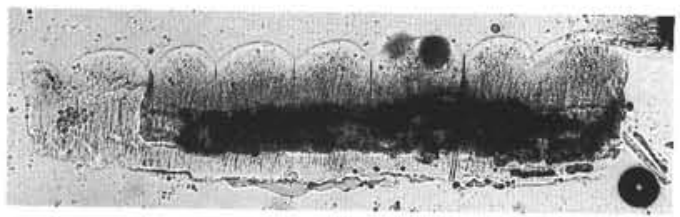

9

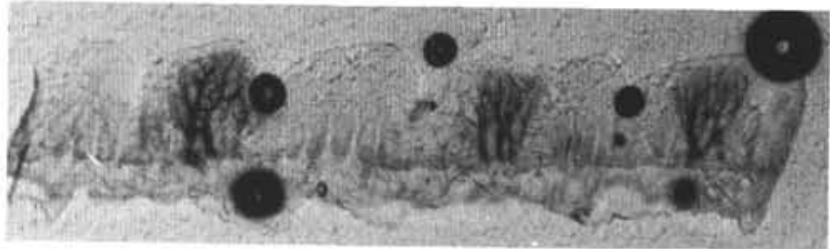

10

\section{1}

Plate 3. 1, 2. Ogee lanceolate, Sample 842B-4H-6, 19-21 cm, 105×; Sample 842B-4H-CC, 105×. 3. Elliptical with line across, Sample 842B-4H-3, $19-21 \mathrm{~cm}, 105 \times$. 4. Two triangles, Sample $842 \mathrm{~B}-4 \mathrm{H}-4,129-131 \mathrm{~cm}, 105 \times$. 5. Rectangular triangular toothed, Sample $842 \mathrm{~B}-4 \mathrm{H}-3,19-21 \mathrm{~cm}, 105 \times$. 6. Small dendritic many radiating lines, Sample $842 \mathrm{~B}-4 \mathrm{H}-2,19-21 \mathrm{~cm}, 210 \times . \quad 7$. Asymmetrical peak wide depression, Sample 842B-4H-6, 19-21 cm, 105x. 8. cf. Two triangles, Sample $842 \mathrm{~B}-4 \mathrm{H}-1,19-21 \mathrm{~cm}, 188 \times$. 9. Rectangular irregularly sawtoothed, Sample 842B-4H-3, 19-21 cm, 105x.

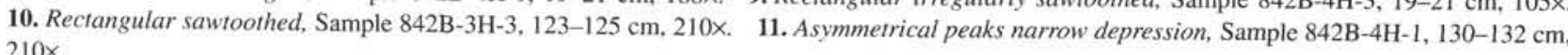




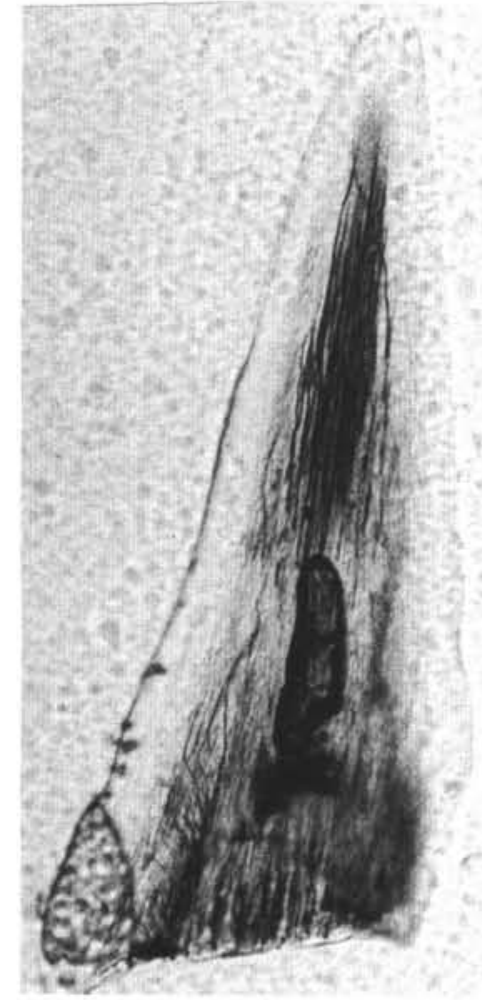

1

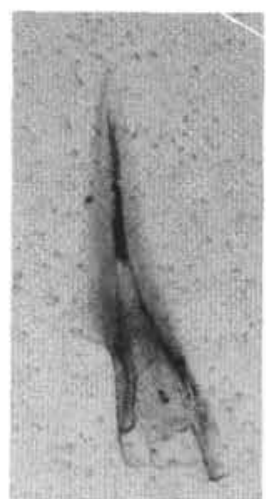

4

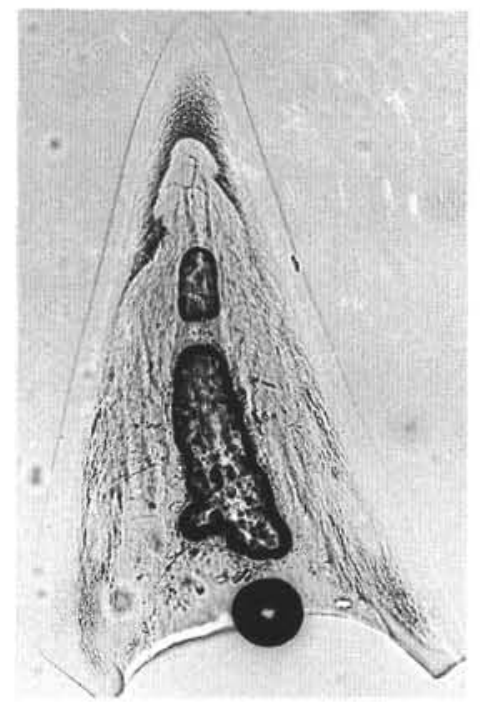

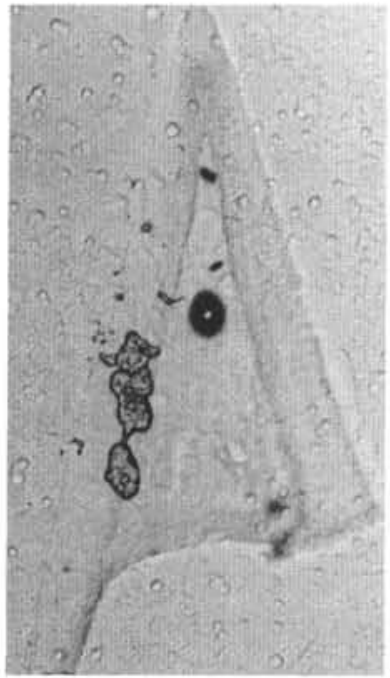

3
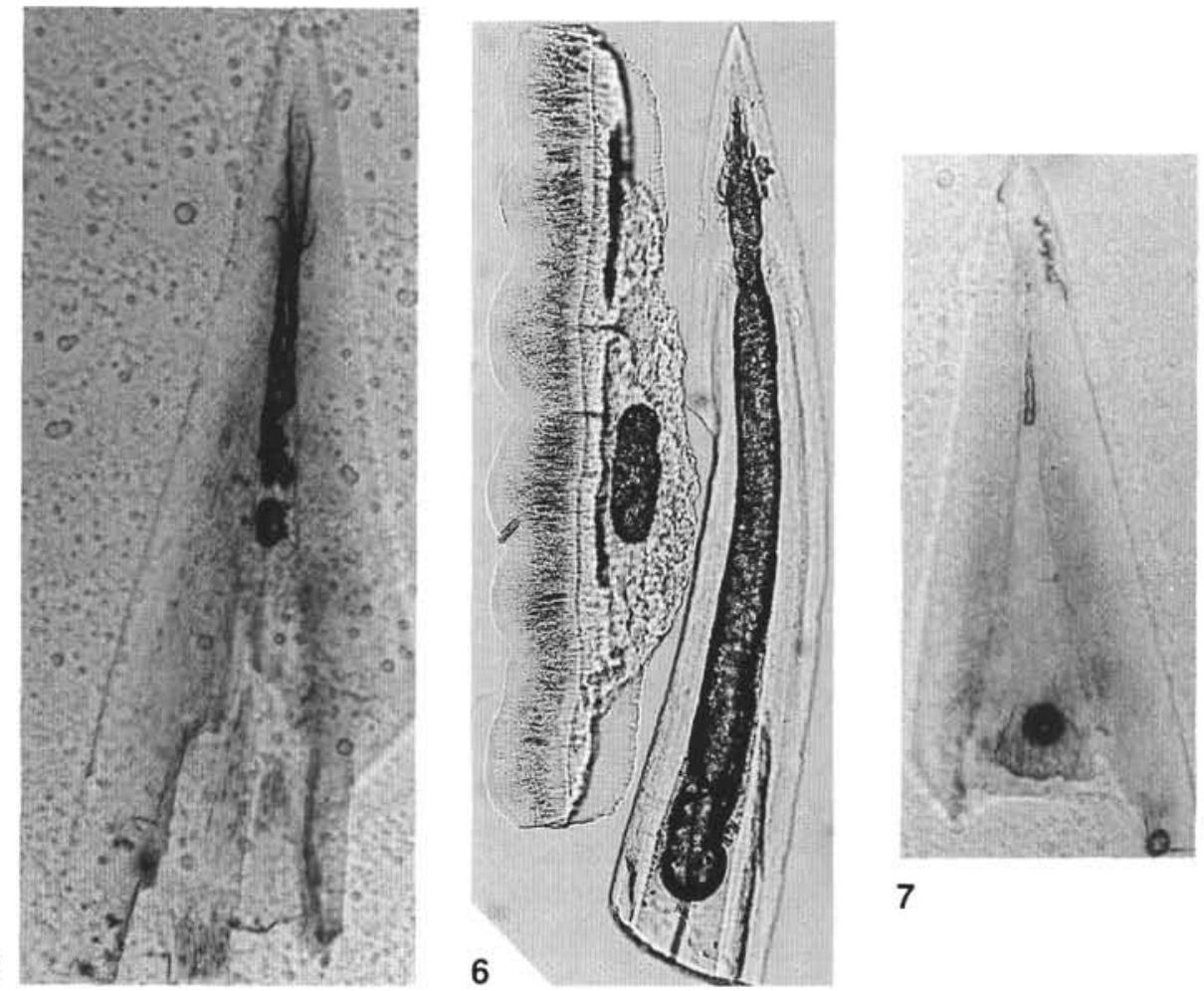

7

Plate 4. 1. Triangle with base angle, Sample $842 \mathrm{~B}-3 \mathrm{H}-7,4-6 \mathrm{~cm}, 206 \mathrm{x}$. 2, 3. Flexed triangle shallow inbase, Sample $842 \mathrm{~B}-4 \mathrm{H}-4,19-21 \mathrm{~cm}, 210 \times$; Sample 842B-3H-5, 27-29 cm, 210x. 4. Flexed triangle 102-112, Sample 842B-3H-3,24-26 cm, 83×. 5. Flexed triangle 120-128, Sample 842B-3H-5, $122-124 \mathrm{~cm}, 165 \times$. 6. Flexed triangle shallow inbase $\geq 120$ (with base attached) and Rectangular irregularly sawtoothed, Sample 842B-4H-3, 130-132 $\mathrm{cm}, 105 \times$. 7. Flexed triangle shallow inbase $\geq 120$, Sample $842 \mathrm{~B}-4 \mathrm{H}-1,19-21 \mathrm{~cm}, 210 \times$. 


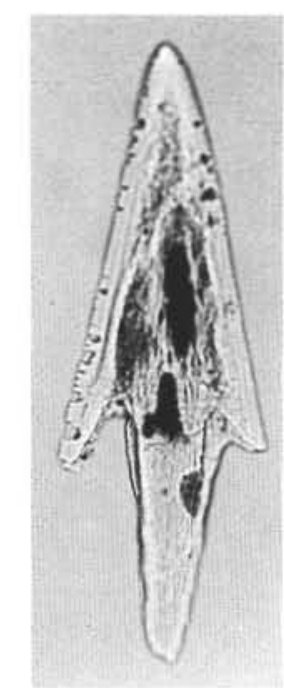

1

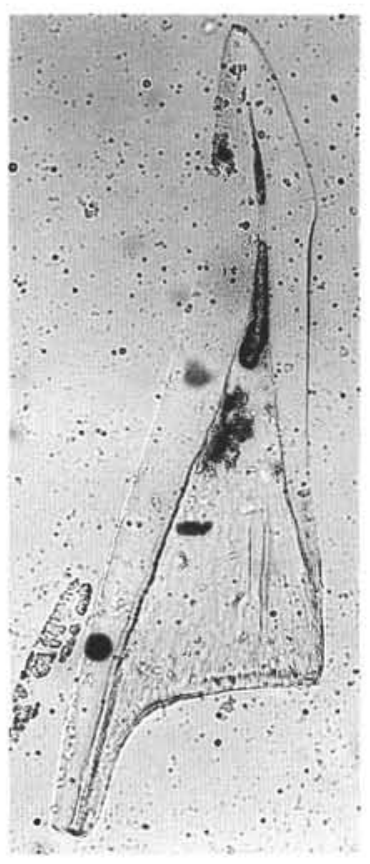

5

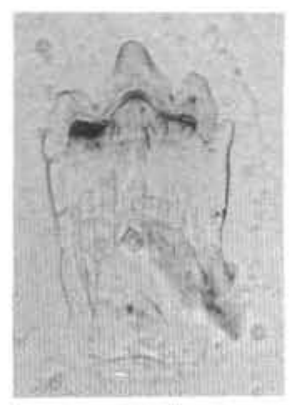

2

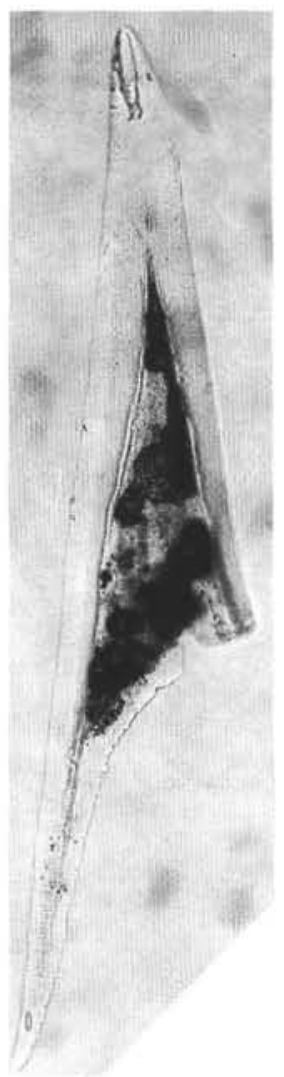

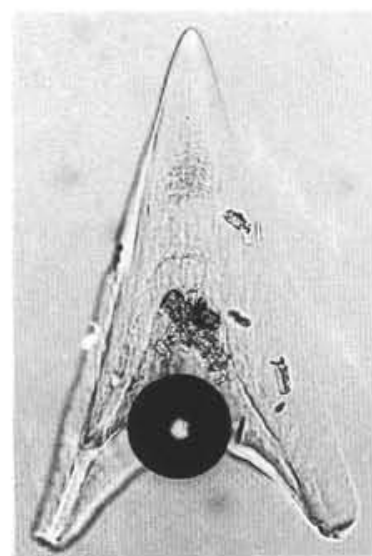

3

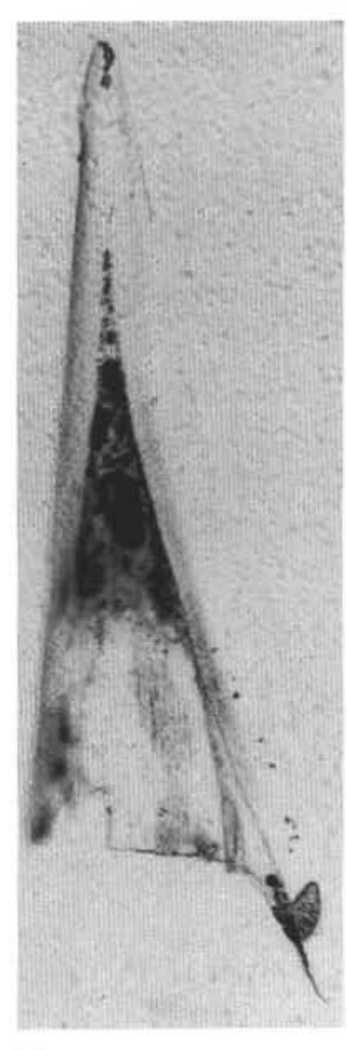

7

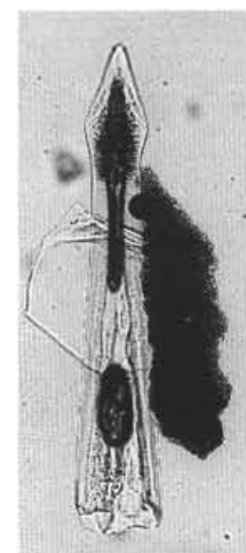

4

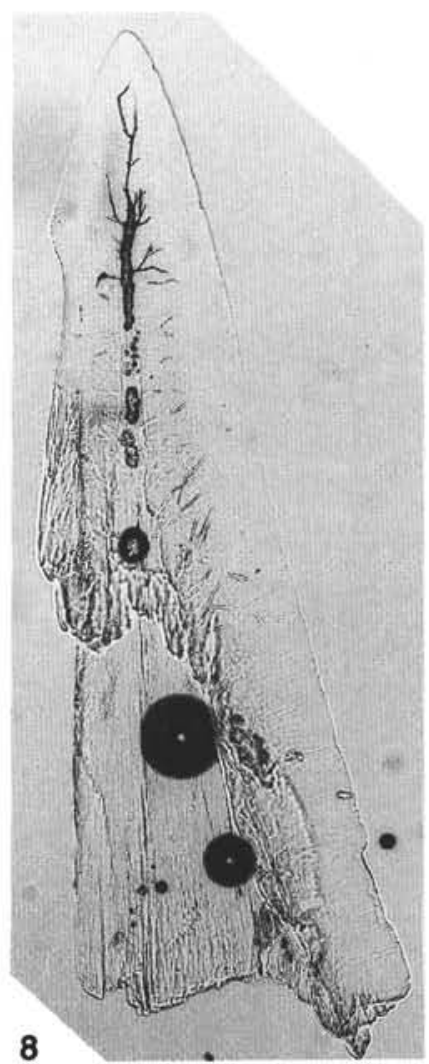

Plate 5. 1. Tanged triangle, Sample $842 \mathrm{~B}-4 \mathrm{H}-6,19-21 \mathrm{~cm}, 105 \times$. 2. Small triangle crenate margin, Sample $842 \mathrm{~B}-4 \mathrm{H}-1,130-132 \mathrm{~cm}, 152 \times$. 3. Triangle curved base, Sample $842 \mathrm{~B}-4 \mathrm{H}-4,129-131 \mathrm{~cm}, 210 \times$. 4. Triangle double wing. Sample $842 \mathrm{~B}-4 \mathrm{H}-7,20-22 \mathrm{~cm}$, 105x. 5. Triangle medium wing, Sample $842 \mathrm{~B}-4 \mathrm{H}-4,129-131 \mathrm{~cm}, 105 \times$. 6, 7. Triangle short wing, Sample $842 \mathrm{~B}-4 \mathrm{H}-\mathrm{CC}, 105 \times$; Sample $842 \mathrm{~B}-3 \mathrm{H}-5,122-124 \mathrm{~cm}, 80 \times$. 8. Triangle broad wing, Sample 842B-4H-3, 19-21 cm, 105x. 


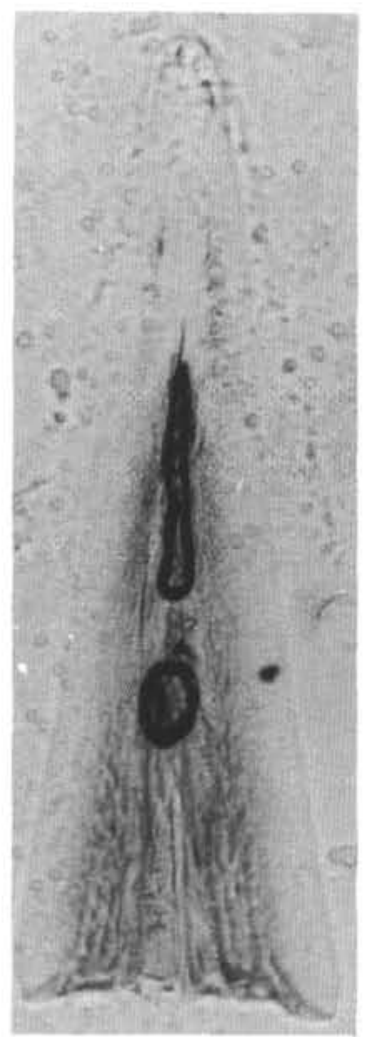

1

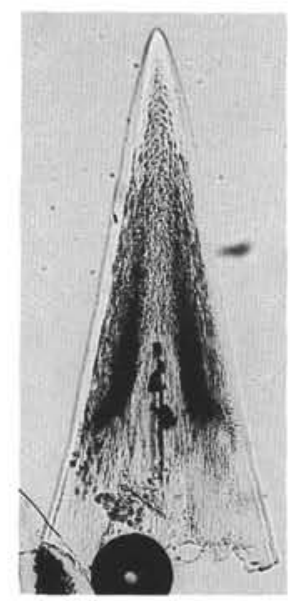

6

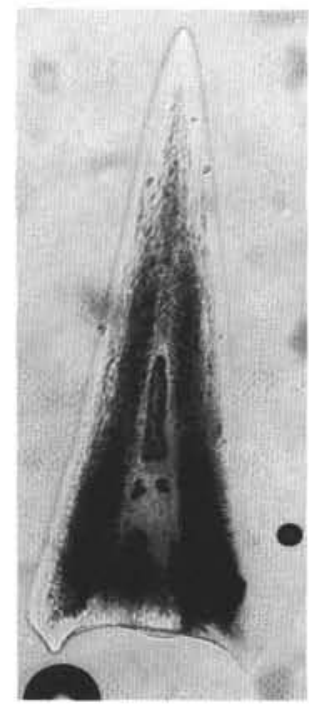

2

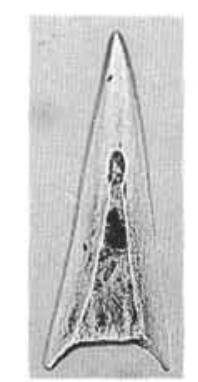

3

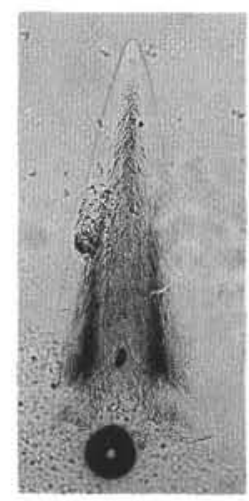

7

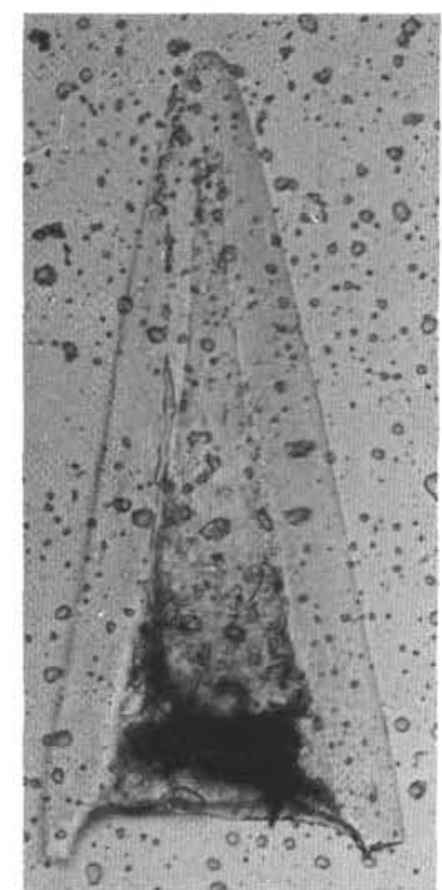

4

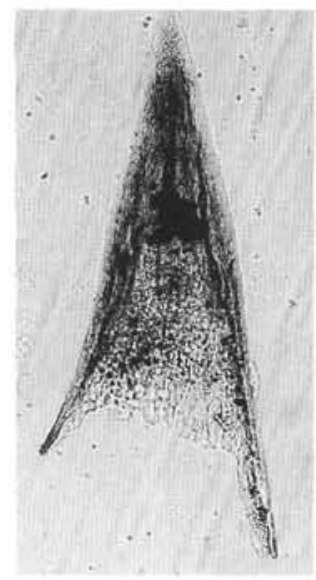

8

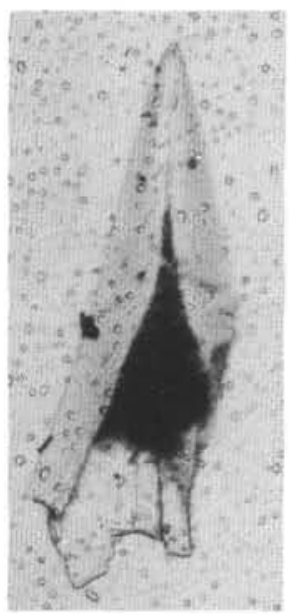

5

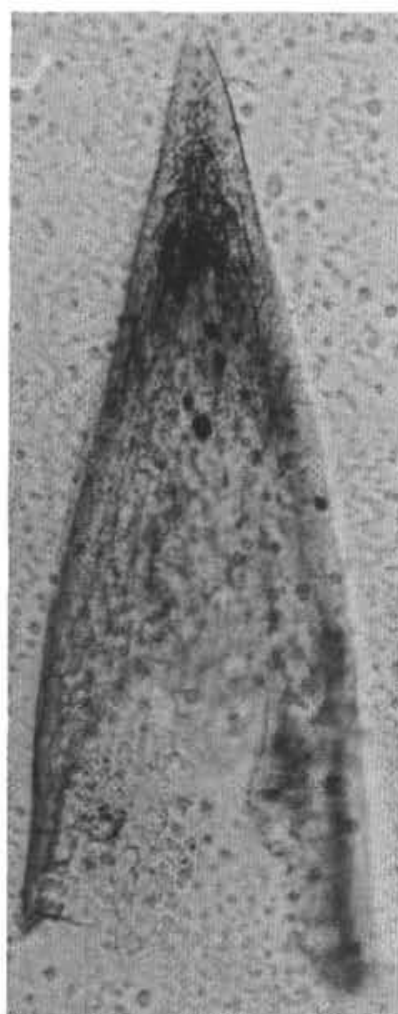

9

Plate 6. 1,3. cf. Triangle pointed margin ends, Sample 842B-4H-1, 130-132 cm, 196×; Sample 842B-4H-3, 19-21 cm, 105×. 2. Triangle pointed margin ends, Sample 842B-4H-CC, 105x. 4. Unnamed form C, Sample 842B-3H-4, 25-27 cm, 202x. 5. Triangle inward angle, Sample 842B-3H-7, 4-6 cm, 74x. 6. Unnamed form E, Sample 842B-4H-4, 19-21 cm, 105x. 7. cf. Pointed triangle short inline, Sample 842B-4H-5, 22-24 cm, 105x. 8,9. Large fibrous triangle, Sample 842B-4H-4, 19-21 cm, 105x; Sample 842B-3H-3, 79-81 cm, 195x. 


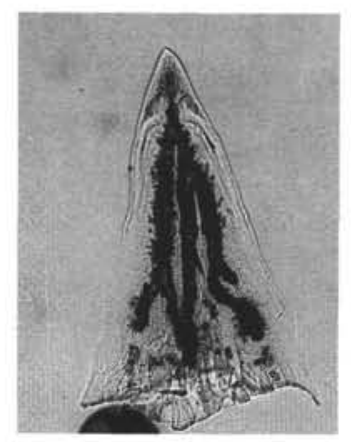

1

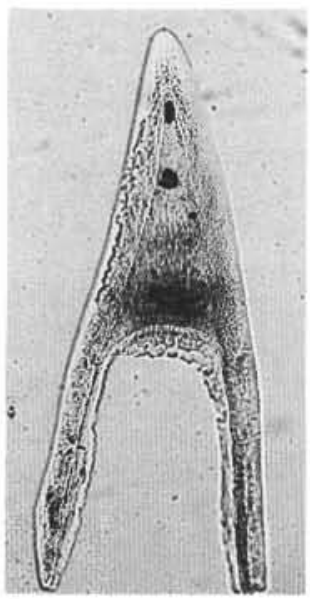

2

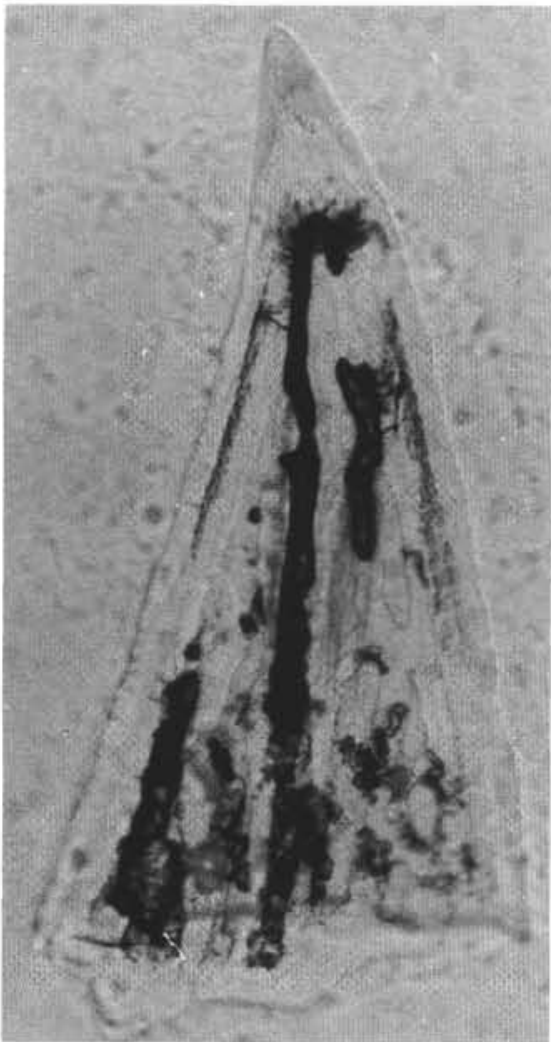

5

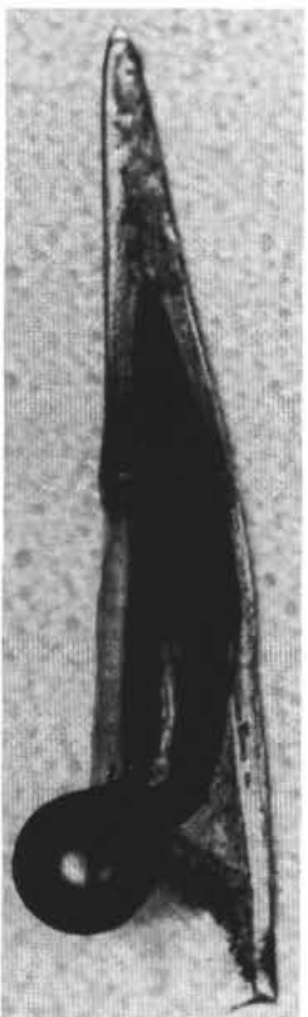

6

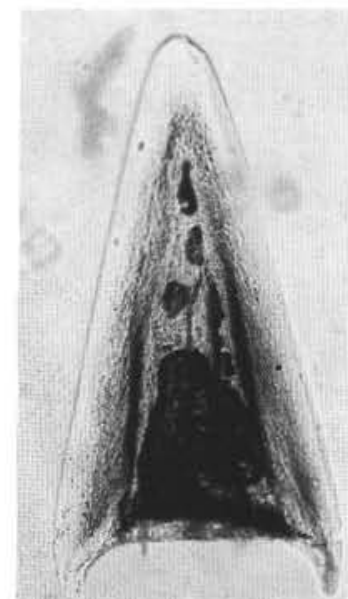

3

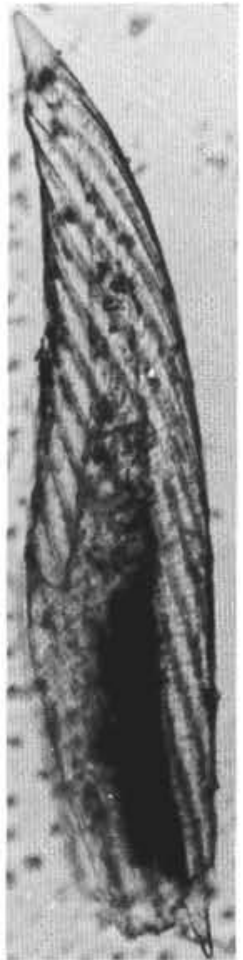

7

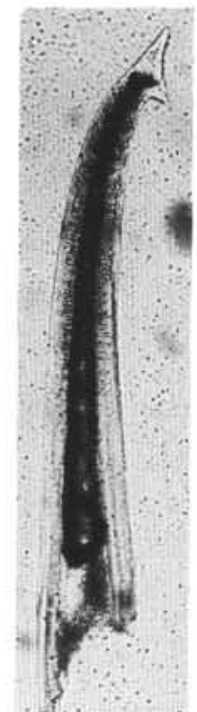

4

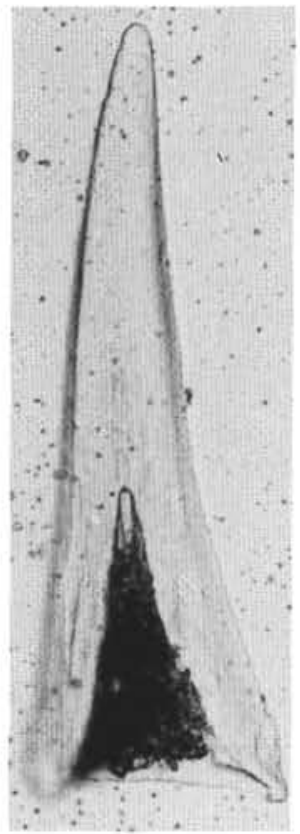

8

Plate 7. 1,5. Triangle with canals, Sample 842B-4H-6, 19-21 cm, 105x; Sample 842B-4H-1, 130-132 cm, 216x. 2. Triangle with high inline apex, Sample 842B-4H-7, 20-22 cm, 105×, 3. Triangle transverse line across, Sample 842B-3H-7, 4-6 cm, 87×; Sample 842B-4H-CC, 105×. 4. Triangle with triangular projection, Sample $842 \mathrm{~B}-4 \mathrm{H}-\mathrm{CC}, 105 \times$. 6. Narrow triangle ragged base, Sample $842 \mathrm{~B}-3 \mathrm{H}-5,122-124 \mathrm{~cm}, 208 \times$. 7. Narrow triangle cross-hachured, Sample 842B-3H-7, 4-6 cm, 202×. 8. cf. Triangle inline halfway, Sample 842B-4H-1, 19-21 cm, 186x. 

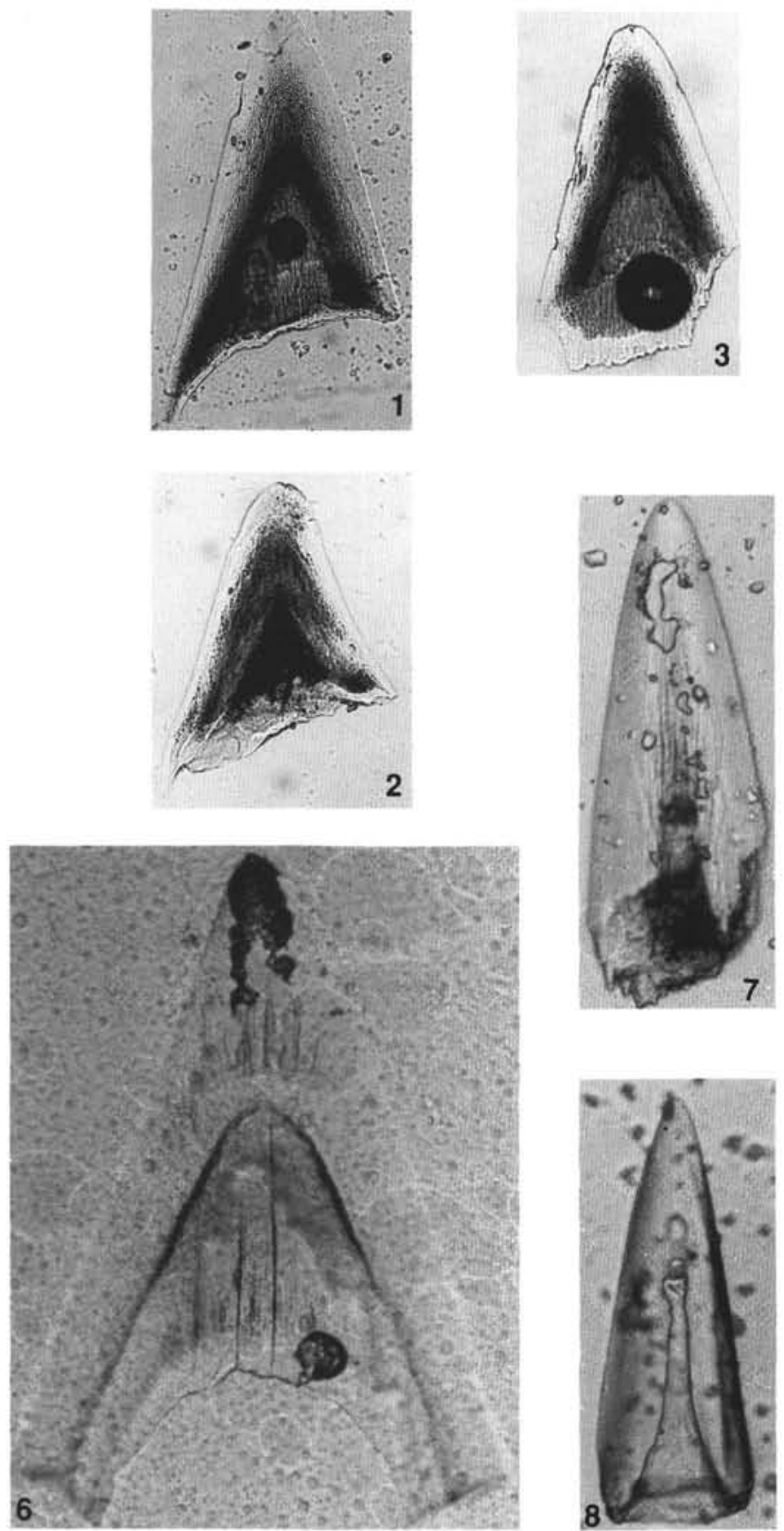

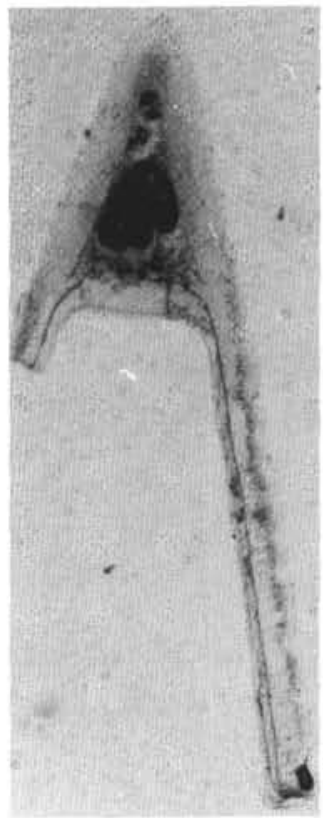

4

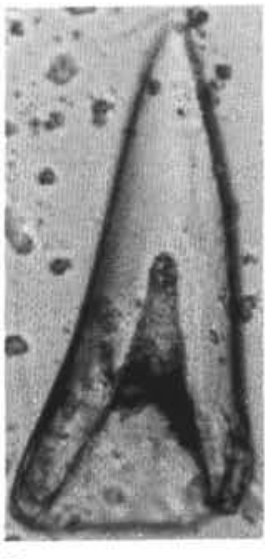

9

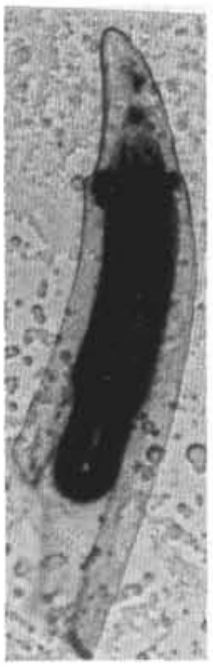

5

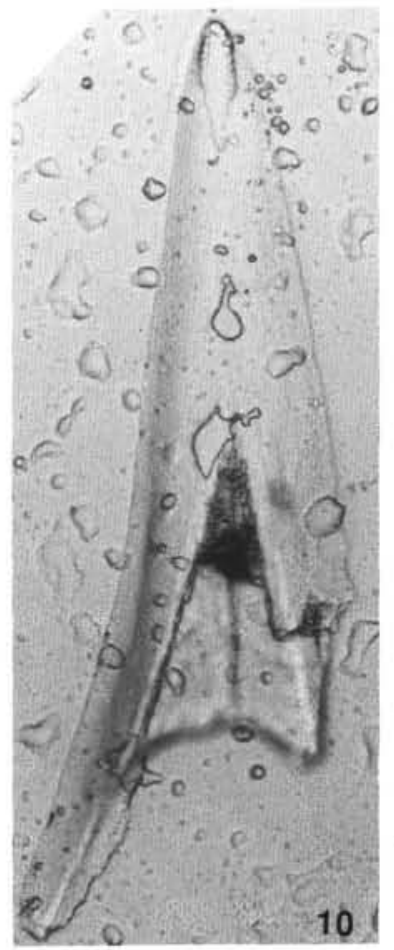

Plate 8. 1-3. Unnamed form A, Sample 842B-4H-7, 20-22 cm, 105x; Sample 842B-4H-4, 19-21 cm, 105x; Sample 842B-4H-5, 129-131 cm, 105x. 4. Triangle crenulate, Sample $842 \mathrm{~B}-4 \mathrm{H}-1,130-132 \mathrm{~cm}, 86 \times$. 5. Short triangle stepped margin, Sample $842 \mathrm{~B}-3 \mathrm{H}-5,27-29 \mathrm{~cm}, 206 \times$. 6. Unnamed form D, Sample 842B-3H-4, 122-124 cm, 205x. 7. Small triangle long striations, Sample 842B-3H-5, 122-124 cm, 220x. 8, 9. Triangle bowed inline, Sample 842B-3H-5, 27-29 cm, 208x; Sample 842B-3H-5, 27-29 cm, 212×. 10. cf. Triangle bowed inline, Sample 842B-3H-7, 4-6 cm, 202×. 


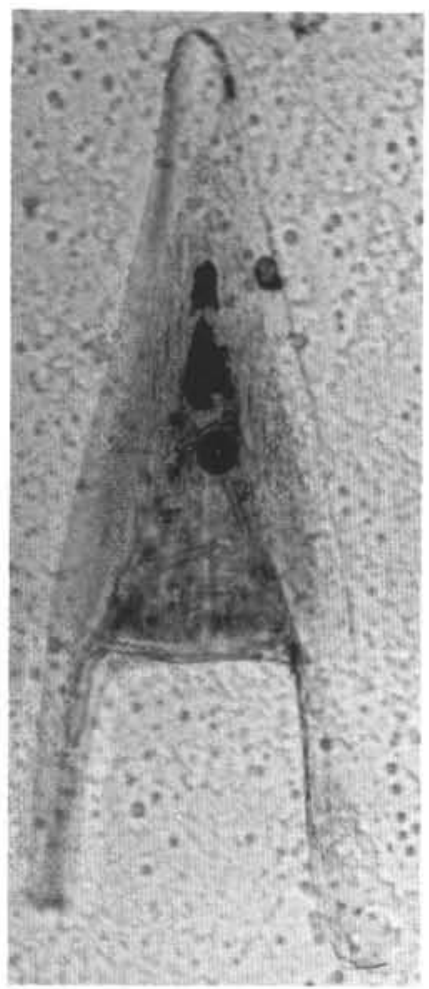

1

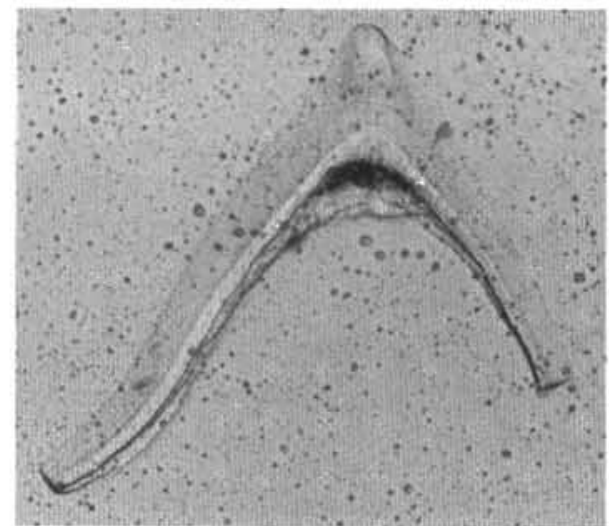

4

2
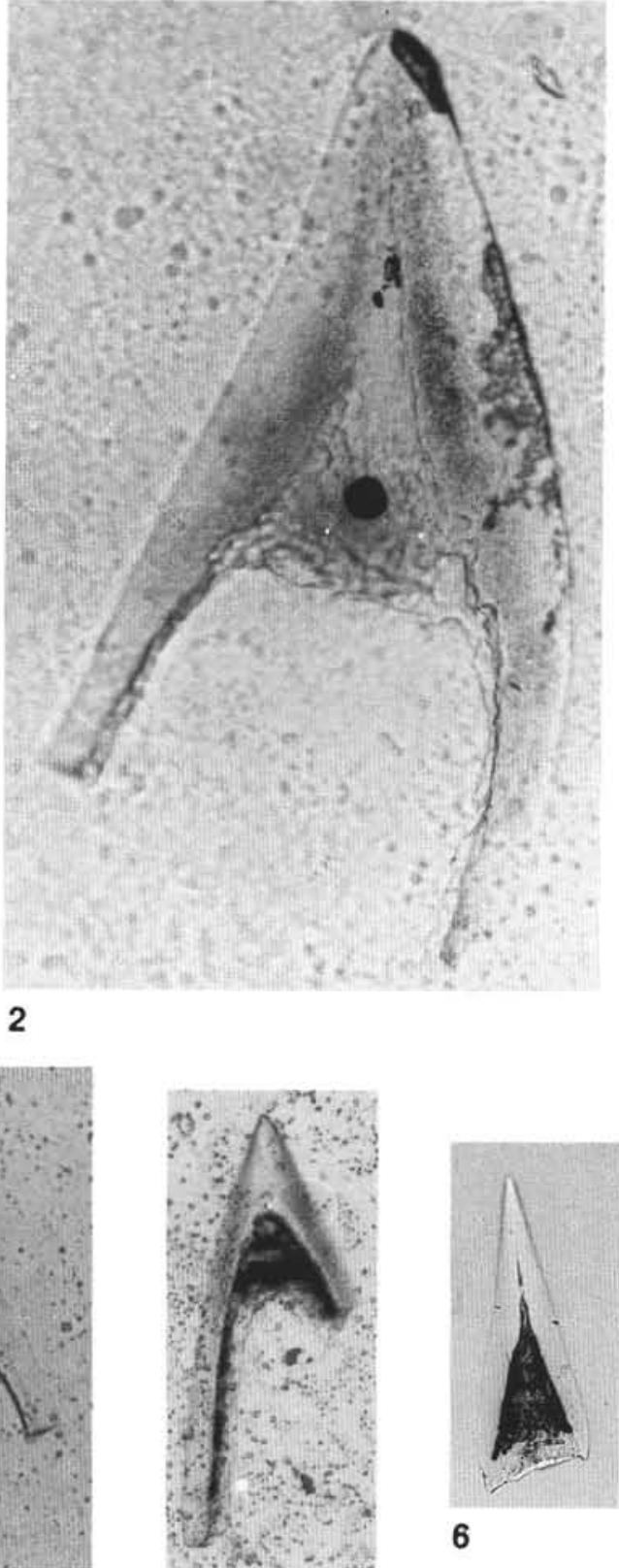

6

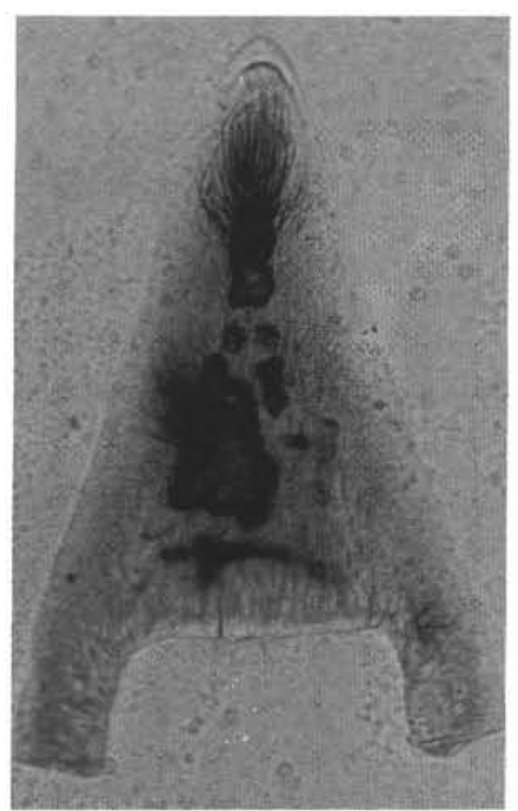

3

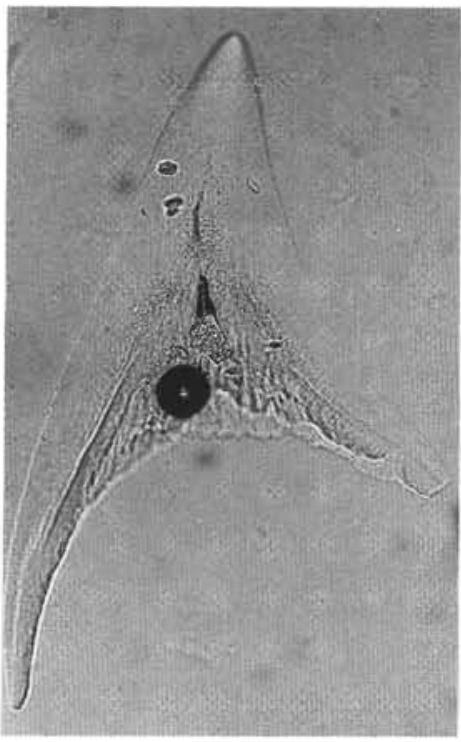

7

Plate 9. 1. Narrow triangle straight inbase, Sample $842 \mathrm{~B}-3 \mathrm{H}-4,122-124 \mathrm{~cm}, 65 \times$ and $204 \times$. 2. Curved triangle pointed margin, Sample $842 \mathrm{~B}-4 \mathrm{H}-1,19-21$ $\mathrm{cm}, 188 \times$. 3. Wide triangle straight inbase, Sample $842 \mathrm{~B}-4 \mathrm{H}-1,130-132 \mathrm{~cm}, 210 \times$. 4, 5. Pointed triangle long margins, Sample 842B-4H-1, 130-132 cm, $200 \times$ (note: pointed apex is broken off); Sample 842B-3H-4, 122-124 cm, 82X. 6. Narrow triangle unequal margins, Sample 842B-4H-3, 19-21 cm, 105x. 7. Curved triangle pointed inline, Sample 842B-4H-6, 19-21 cm, 105×. 

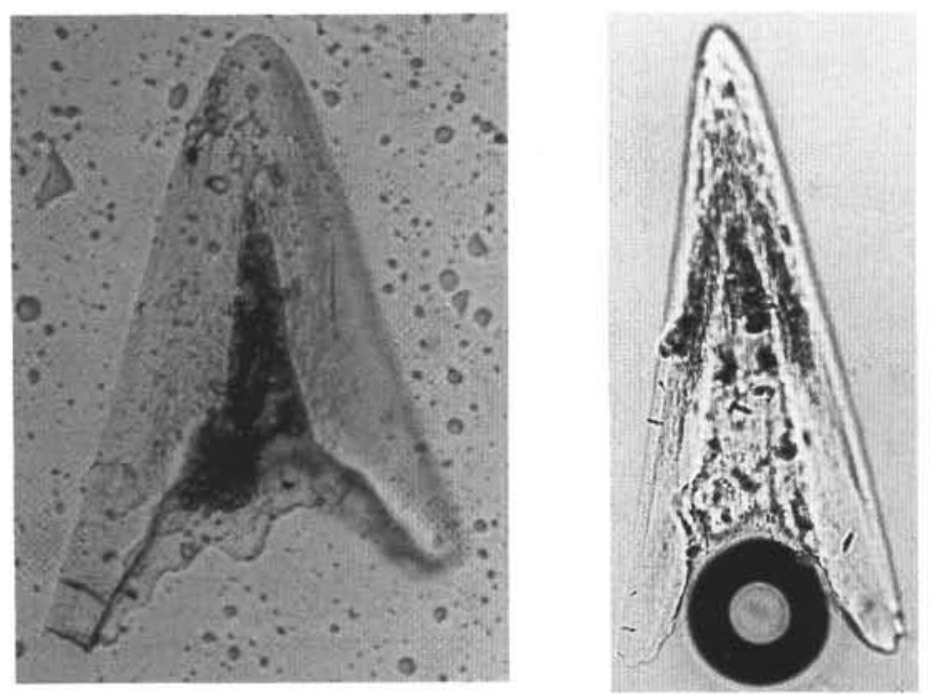

2

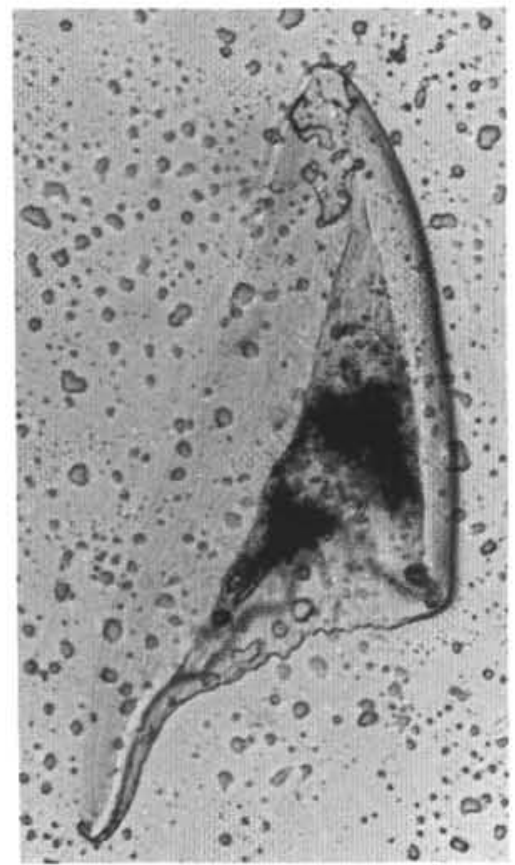

5

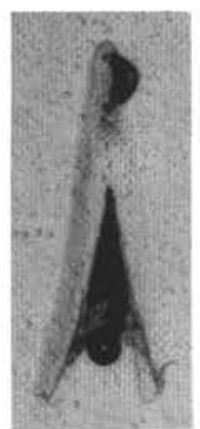

3

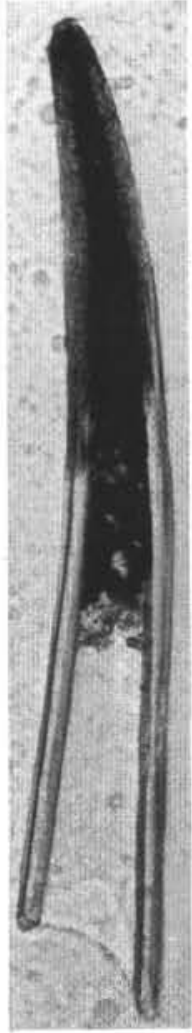

7

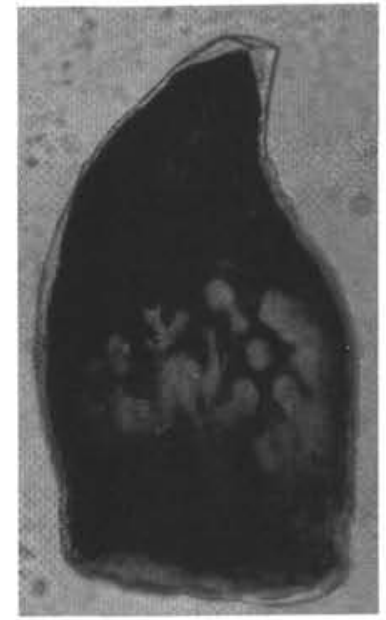

4

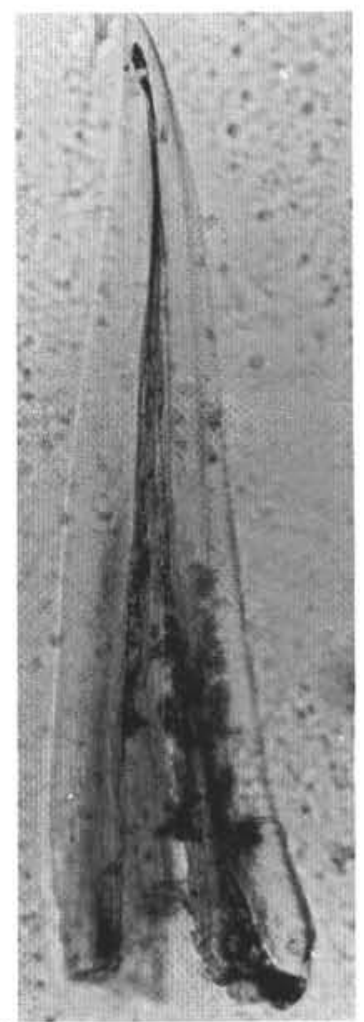

8

Plate 10. 1. Triangle sinuous inline, Sample $842 \mathrm{~B}-3 \mathrm{H}-3,123-125 \mathrm{~cm}, 206 \times$. 2, 3. Triangle concave base, Sample 842B-4H-4, 129-131 cm, 210×; Sample 842B-3H-3, 79-81 cm, 64×. 4. Stippled triangle, Sample 842B-4H-1, 130-132 cm, 210x. 5. Triangle hooked margin, Sample 842B-3H-6, $27-29 \mathrm{~cm}, 230 \times$. 6. Triangle sigmoid, Sample 842B-3H-6, 27-29 cm, 215×. 7. Long triangle thin wall, Sample 842B-4H-1, 19-21 cm, 188X. 8. Triangle long inline, Sample $842 \mathrm{~B}-3 \mathrm{H}-7,4-6 \mathrm{~cm}, 148 \times$. 